\title{
Is it a fallacy to believe in the hot hand in the NBA three-point contest? ${ }^{\text {मै }}$
}

\author{
Joshua B. Miller ${ }^{\text {a, }{ }^{* * *}}$, Adam Sanjurjo ${ }^{\text {b, }{ }^{* * *}}$

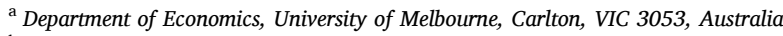 \\ ${ }^{\mathrm{b}}$ Fundamentos del Análisis Económico (FAE), Universidad de Alicante, San Vicente 03690, Spain
}

\section{A R T I C L E I N F O}

\section{JEL classification:}

C12

C14

C91

C93

D03

Keywords:

Hot hand fallacy

Hot hand effect

\begin{abstract}
A B S T R A C T
The NBA Three-Point Contest has been considered an ideal setting to study the hot hand, as it showcases the elite professional shooters that hot hand beliefs are typically directed towards, but in an environment that eliminates many of the confounds present in game action. We collect 34 years of NBA Three-Point Contest television broadcast data (1986-2020), apply a statistical approach that improves on those of previous studies, and find considerable evidence of hot hand shooting in and across individuals. Our results support fans' and experts' widely held belief in the hot hand among NBA shooters.
\end{abstract}

\section{Introduction}

On January 23, 2015, professional basketball player Klay Thompson, of the NBA's Golden State Warriors, hit 13 consecutive shots, 9 of which were taken from long distance ("3-point" range). Just three weeks later, his teammate, Stephen Curry, hit 13 consecutive shots in the all-star weekend 3-point shooting contest. In each case, teammates (and other observers) universally described the player as being "hot," i.e. in a state of elevated shooting ability. Consistent with these beliefs, the evidence suggests that players are more likely to allocate the ball to a teammate who they believe has a hot hand. ${ }^{1}$ As an example, in a game that occurred within weeks of Thompson's performance, LeBron James, arguably the world's best player at the time, spontaneously changed the design of plays during a time stoppage in order to continue targeting his teammate Kevin Love; when asked about this after the game, James replied: "He had the hot hand, I wanted to keep going to him."2

The academic consensus, however, over the better part of the last three and a half decades, has been that the beliefs of these players is clear evidence of a "massive and widespread cognitive illusion," by which people perceive an atypical clustering of successes when there is none (Kahneman, 2011). Accordingly, the hot hand fallacy has been considered, more generally, as a candidate explanation for

\footnotetext{
Financial support from the Department of Decision Sciences at Bocconi University and the Spanish Ministry of Science and Innovation (Grant PID2019-108193GB-I00) is gratefully acknowledged.

E-mail addresses: Joshua.Benjamin.Miller@gmail.com (J.B. Miller), sanjurjo@ua.es (A. Sanjurjo).

*** Both authors contributed equally, with names listed in alphabetical order.

1 See Neiman and Loewenstein (2011); Rao (2009a); Cao (2011); Attali (2013); Bocskocsky et al. (2014); Aharoni and Sarig (2011).

${ }^{2}$ See the ESPN article https://www.espn.com/blog/cleveland-cavaliers/post/_/id/435 posted on Feb 9, 2015.
} 
various puzzles and behavioral anomalies identified in a variety of domains, including financial markets, ${ }^{3}$ sports wagering, ${ }^{4}$ casino gambling, ${ }^{5}$ and lotteries. ${ }^{6}$

However, a large statistical bias in the measures used in the canonical study of Gilovich et al. (1985) has recently been observed (Miller and Sanjurjo, 2018). Once the bias is corrected for in Gilovich et al. (1985)'s analysis, the results of their controlled shooting experiment reverse, yielding strong evidence of hot hand shooting. Further, this evidence has now been replicated across several controlled shooting experiments (Miller and Sanjurjo, 2018; 2014).

While controlled shooting experiments may be critical to the scientific study of the hot hand, because they eliminate myriad confounds that are present in game environments, ${ }^{7}$ a potential limitation is that it is difficult for them to simulate certain features of a professional game environment, such as professional shooters, sizeable incentives, and large crowds. To the extent that the presence or absence of such features could lead to systematic differences in hot hand shooting, it would be advantageous to preserve them, particularly because it is precisely the shooting of well-known professionals that peoples' hot hand beliefs are commonly based on.

A setting that combines desirable features from both shooting environments is the annual NBA all-star weekend Three Point Contest. In particular, it offers: (i) a rough approximation of the type of shooting found in NBA games, with the features mentioned above preserved, and (ii) several of the crucial features of a controlled shooting experiment, so that many of the confounds present in games are eliminated. It has been considered "an ideal situation in which to study the hot hand" (Thaler and Sunstein, 2008).

In the contest, a group of the NBA's best shooters are selected to compete against one another in multiple rounds. In the first round each shooter is given 60 seconds to shoot 25 balls -5 at each of 5 racks that span the three point line, and are roughly equally spaced. More successful shooters then advance to compete in additional rounds, in which the task is the same as in the first. As in games, players shoot on an NBA court, under high stakes, and in front of a large crowd and television audience. Unlike in games, each shot is taken from (roughly) the same distance, in close temporal proximity, and without many of the strategic confounds present in games (shot distance, variable defensive pressure, offensive and defensive play calls, etc.).

Koehler and Conley (2003) study four years of NBA Three Point Contest data (1994-1997) and, despite the well-known beliefs of players, coaches, and fans, find no evidence of a hot hand in performance. As a result, their findings have long stood as a prominent replication of the canonical results of Gilovich et al. (1985).

In this study we instead collect thirty-four years (1986-2020) of NBA Three Point Contest data from television broadcasts, and apply an empirical approach that is: (i) more powered, (ii) contains an improved set of statistical measures, and (iii) corrects for the strong downward bias in estimates of the hot hand effect that is present in previous analyses.

In contrast with the results of Koehler and Conley (2003), we observe a significantly large number of hot hand shooters. In addition, despite heterogeneity in the size (and sometimes even the sign) of the hot hand effect across individual shooters, in a pooled analysis players on average shoot a considerable +5 to +9 percentage points better when on a streak of hits. Finally, by repeating our analysis on miss streaks we can identify whether observed streak shooting is due to the hot hand or the cold hand. We find substantial evidence of hot hand shooting and little evidence of cold hand shooting.

The discovery of considerable hot hand shooting in elite professional shooters supports the widespread belief in the hot hand by experts and casual fans alike, and corroborates the recent finding of hot hand shooting across all extant controlled shooting experiments (with professional and non-professional shooters).

\section{Data}

\section{NBA Three Point Contest}

The annual NBA all-star weekend Three Point Contest began in 1986, with the following format. Eight of the NBA's best three point shooters are selected to participate in a tournament consisting of three rounds. In each round, each participant has the opportunity to shoot 25 times -5 times from each of 5 rack locations which span the three point line, and are roughly equally spaced. The three point line is 22 feet $(6.7 \mathrm{~m})$ from the basket in the corners of the court, a distance that increases along a 14 foot ( $4.3 \mathrm{~m})$ straight line that runs parallel to the sidelines on either side. At the 14 foot mark the three point line becomes an arc 23.75 feet ( $7.2 \mathrm{~m}$ ) from the basket. The shooter has $60 \mathrm{~s}$ to complete as many of these 25 shots as possible. All misses are worth zero points, while hits (makes) are worth either

\footnotetext{
3 See, e.g. Rabin and Vayanos (2010), Malkiel (2011), De Long et al. (1991), Loh and Warachka (2012), Kahneman and Riepe (1998), De Bondt (1993), and Barberis and Thaler (2003).

4 See, e.g. Camerer (1989), Durham et al. (2005), Paul and Weinbach (2005), Brown and Sauer (1993), Sinkey and Logan (2013), Avery and Chevalier (1999), Arkes (2011), and Lee and Smith (2002).

5 See, e.g. Croson and Sundali (2005), Sundali and Croson (2006), Narayanan and Manchanda (2012), Smith et al. (2009), and Xu and Harvey (2014).

6 See, e.g. Galbo-Jørgensen et al. (2016), Guryan and Kearney (2008), Yuan et al. (2014).

7 For example, Rao (2009a) and Bocskocsky et al. (2014) have shown that omitted variable bias in analyses of game data leads to underestimation of the hot hand. While some strategic confounds may be inherently difficult to control for (Green and Zwiebel, 2018; Nalebuff, 1987; Aharoni and Sarig, 2011), one can add partial controls for factors such as shot distance and defensive pressure in order to mitigate this issue. However, doing so necessitates greater pooling of data across players and games (sometimes multiple seasons), and thus additional partial controls. Pooling, in turn creates a separate fundamental challenge to detecting the hot hand: absence of hot hand shooting in the pooled result could be driven by a mixture of hot shooters and the opposite. Another challenge is that in NBA games the players select when and under what circumstances they shoot, so, for example, a hot player may have private motivations to take an unfavorable shot rather than pass to a teammate who is open due to the extra attention the hot player attracts.
} 
one point (first four balls on each rack) or two points (fifth ball on each rack). The four players with the highest point totals in round one advance to round two. ${ }^{8}$ Those two players with the highest point totals in round two advance to the finals, and the player with the highest point total in the finals wins the contest $(\$ 10,000)$-along with the indirect benefits that are likely to result from the strengthening of one's reputation as an elite shooter.

The format of the contest has experienced some minor changes over the years. From 2003 to 2013 there were six participants, rather than the usual eight, and in 2019 there were ten. The number of rounds has decreased from three (1986-1998) to two (2000-2020). ${ }^{9}$ The distance of the three point line changed for the 1995-1997 contests to 22 feet (6.7 m) along the entire arc, and the number of two-point balls changed from 5 to 9 beginning in $2014 .{ }^{10,11}$ In 2020, the time per round was increased from 60 to 70 s to allow for the addition of two longer distance shots, each worth three points. These "Dew Zone" shots were taken from each side of the center rack at a distance of 29.9 feet $(9.1 \mathrm{~m})$ from the basket. Prize money has increased over the years, with the winner receiving $\$ 50,000$ in the most recent contest.

\section{Data collection}

We collect video records from every annual NBA Three Point Contest telecast from 1986 (the first) to 2020, though two of the telecasts were incomplete. ${ }^{12}$ This gives us shots from 34 contests, and 123 players, with a median of 50 shots per player ( 37 players take at least 100 shots).

Data for each shooting round was coded from the original televised video footage, and consisted of hits (1), misses (0), and the time elapsed between shots. Shots that were taken after the time in a round had expired were not included. In a few rare cases, players stepped on the line, but were typically unaware of this until after the round had ended. We included these shots, though the contest did not, as we judged the shots to be taken under near identical conditions (a few centimeters difference) as shots in which the player was just slightly behind the line. ${ }^{13}$ The summary statistics of the coded data were cross-validated with Table 1 of Koehler and Conley (2003), which summarizes individual player performance in the four contests held from 1994 to 1997, with the table successfully reproduced up to minor discrepancies. ${ }^{14}$

\section{Empirical strategy}

We first briefly describe the empirical approach and results of Koehler and Conley (2003)'s analysis of the NBA's Three Point Contest, and then discuss some of its important limitations. Then we describe our empirical approach, and detail its relative advantages.

\section{Koehler and Conley's analysis}

Koehler and Conley (2003)'s analysis consists of two types of tests, each defined in the canonical study of Gilovich et al. (1985): (i) a "conditional probability" test, and (ii) a runs test.

The conditional probability test provides the most intuitive measure of hot hand shooting, as it is used to test whether a shooter's probability of success is higher (immediately) following a streak of hits than it is following a streak of misses. Koehler and Conley (2003) find that players' average shooting percentage following streaks of three hits (57.3\%) is similar to their average shooting percentage following streaks of three misses (57.5\%). Further, Koehler and Conley (2003) find that just as many players have a relatively lower shooting percentage following streaks of three hits (relative to the percentage following streaks of three misses), as the

\footnotetext{
${ }^{8}$ In the case of a tie between two shooters in contention to move to the next round, 24 s elimination rounds, with the rules otherwise the same, are conducted until one participant has a strictly lower score than the other, in which case he is eliminated.

9 There was no contest in 1999, due to an NBA lockout.

10 In particular, beginning in 2014, each player selects one of his five racks to have its four one-point balls replaced by two-point balls.

11 Also, the amount of seconds given to shooters in tie-breaking elimination rounds has varied from 24 , to 30 , to 60 seconds.

12 In particular, we were unable to obtain complete video records for the first two rounds of the first contest (1986; 14 rounds lost) given that the producers cut out most of the shots from the telecast. Also, we were unable to obtain the first round records for six shooters in the 1993 contest. As mentioned in Footnote 9, there was no contest in 1999.

13 An even rarer occurrence was when the video footage did not clearly display the outcome of a shot, as in the case of a momentary obstruction in front of the camera. In these few cases the combination of the announcers' audio data, the player's final point total, and the televised score-sheet (in the years that this was available) was always enough to confidently infer the outcome of the shot.

14 The data from Koehler and Conley (2003) no longer exists. We thank Jonathan J. Koehler for searching his records for us. We record the following data from 1994 to 1997, which Koehler and Conley omit: (i) a three-way 30 s tie-break round between Steve Kerr, George McCloud, and Dana Barros (1996, first round), (ii) two successive tie-breaks between Eric Murdock and Dana Barros (1994, first round), (iii) a 25-shot round from Steve Kerr (we are unsure which round was omitted, as the authors do not document rounds). In addition, we found discrepancies with Koehler and Conley's Table 1 for 4 rows of players (Miller, Rice, Legler, Scott); our records differed by 1 or 2 shots, which could be attributed to different coding rules for shots taken while a player's foot is on the line. For the remaining rows, all data and statistics match. The NBA's official online record of the three point contest scores is no longer available.
} 
other way around. ${ }^{15}$

The runs test is a common test of whether an atypical clustering of like outcomes occurs in a sequence. A run is defined as an uninterrupted block of consecutive hits (misses) that begins and ends with either: (i) a miss (hit) or (ii) the beginning/end of the sequence. The runs test compares the total number of runs observed in the sequence of shots to the number expected under the null hypothesis that the player has a fixed probability of success (i.e. is an i.i.d. Bernoulli shooter). The hot hand (or cold hand) hypothesis predicts the observation of fewer total runs than expected. ${ }^{16}$ Koehler and Conley (2003) find that only two out of 23 shooters exhibit significantly fewer runs than expected by chance. ${ }^{17}$

An important limitation of Koehler and Conley (2003)'s analysis can be found in their conditional probability test, which uses the difference between a player's shooting percentage following streaks of hits vs. misses as an estimator of the difference in the corresponding probabilities. Miller and Sanjurjo (2018) demonstrate that this estimator is biased, and that the bias is large for sample sizes often used in empirical work. ${ }^{18}$ For example, in a 25 -shot round of the contest a consistent shooter with an i.i.d. .5 probability of success is expected to have a shooting percentage that is 26 percentage points lower following streaks of three hits than it is following streaks of three misses; in a 100 shot session the difference is expected to be 8 percentage points. ${ }^{19}$ Thus, under the assumption of no hot hand, we should expect the majority of players to exhibit a lower shooting percentage following streaks of hits than they do following streaks of misses.

\section{Empirical Approach}

We follow the empirical approach of Miller and Sanjurjo (2014), which: (i) increases statistical power relative to those of previous studies, ${ }^{20}$ (ii) uses an improved set of statistical measures, (iii) estimates the effect of hit streaks on players' shooting percentage without bias, (iv) separates hot hand from cold hand shooting, and (v) facilitates comparison of the present findings with those of controlled shooting experiments.

In addition to performing a conditional probability test similar to that of Koehler and Conley (2003), we use a set of four test statistics that reflect different conceptions of hot hand shooting: (1) the hit streak frequency $\left(H_{F}\right)$ measures the relative frequency of shots taken immediately following three or more consecutive hits (made shots), i.e. "how often a shooter is on a streak," (2) the hit streak momentum $\left(H_{M}\right)$ measures the relative frequency of hits on shots taken immediately following three or more consecutive hits (made shots), i.e. "how well a shooter performs when on a streak," (3) the hit streak length $\left(H_{L}\right)$ measures the length of the longest run of hits, which was previously used by Wardrop (1999), and (4) the standard runs statistic $(R) .{ }^{21}$ For each of our hit streak statistics $H_{F}, H_{M}$, and $H_{L}$ we provide an analogously defined miss streak statistic $M_{F}, M_{M}$, and $M_{L}$. The separate treatment of streaks in hits, and misses, provides an important methodological advantage over previously used measures of "streak shooting" and "momentum" that do not distinguish between hot hand and cold hand shooting.

The statistical test procedure begins with the null hypothesis $\left(H_{0}\right)$ that a player does not get hot (or cold)—i.e. that the player's sequence of shot outcomes are generated by an independent Bernoulli process with a fixed (unknown) probability of success. Because the shot outcomes are exchangeable under $\mathrm{H}_{0}$-i.e. all orderings of the shot outcomes are equally likely-the exact sampling distribution of any test statistic can be constructed by calculating the value of the test statistic for each (unique) permutation of the player's shots, then building the histogram with these values. We approximate the sampling distribution of each statistic using 25,000 random permutations. Unlike the analogous tests of Gilovich et al. (1985) and Koehler and Conley (2003), this approach is unsusceptible to bias because the finite sample properties of the test statistic are reflected in its sampling distribution.

\footnotetext{
15 Koehler and Conley (2003) also find that just as many players shoot relatively better following streaks of three misses (when compared to their overall shooting percentage), as the other way around.

16 For example, in the 10 shot sequence 1101000111 there are five runs: "11," "0," " 1 ,"“ 000 ," and " 111 ," whereas in the "streakier" shot sequence 1111110000 there are only two: "111111" and "0000."

17 If a player had more than one 25 shot round, the authors construct a single long sequence of shots, as if all the shots were taken successively within a single round. They also conduct a separate runs test for each round and find that the average number of runs (12.5) is close to the expected number (13).

18 The estimator has also been shown to be equivalent to the runs test for the special case of streaks of length one (Miller and Sanjurjo, 2014).

19 The average player shoots 60 shots in Koehler and Conley (2003), so the bias is slightly less than 16 percentage points for the average player in their sample.

20 See the Appendix of Miller and Sanjurjo (2014).

21 Possible mechanisms for hot hand shooting are: (i) shifting to a relatively higher performance state (due to, for example, internal or external factors leading to increased focus, attention, or motor control (Churchland et al., 2006; Kahneman, 1973; Csikszentmihalyi, 1988)), (ii) performing better following recent success (due to, for example, positive feedback leading to increased confidence (Bandura, 1982)), or some combination of (i) and (ii). Because either mechanism involves a player temporarily shooting with a higher probability of success, we should expect hot hand shooting to translate into a tendency for hits to cluster. Our statistics measure the tendency of a player to produce hit streaks, and represent the patterns typically associated with hot hand shooting. For a discussion of the patterns, see Gilovich et al. (1985); Tversky and Gilovich (1989); Gould (1989); Wardrop (1999). For a summary account see Reifman (2012). For a power analysis of the hit streak statistics across several models of hot hand shooting, see Miller and Sanjurjo (2014). We leave the task of determining which model of hot hand shooting best fits the data for future work.
} 
Our hit streak statistics are non-negative, and the permutation approach reveals that they have skewed sampling distributions. As a result, in our analysis of each of these statistics we employ and report the $p$-value associated with the relevant tail of the sampling distribution. For the statistics $H_{F}, H_{M}$, and $H_{L}$ this is the right tail, while for $R$ and the minimum p-value test statistic (defined below) this is the left tail. ${ }^{22}$ These are the relevant tails because the alternative hypothesis of hot hand shooting establishes a clear ex ante directional prediction for these statistics. In particular, the hot hand "fallacy" is based on the belief that players shoot better when on a streak of success, not worse (as in the gambler's fallacy). ${ }^{23}$ For our conditional probability test, and the panel data regressions covered in Section 4.3, we follow standard convention by reporting the $p$-values that correspond to two-sided tests.

We address multiple testing issues across shooters and statistics by using: (i) binomial tests on the number of significant results of a given statistic across all shooters, (ii) a summary test statistic equal to the minimum p-value across the three (correlated) hit (miss) streak test-statistics (MacKinnon, 2009; Westfall and Young, 1993). ${ }^{24}$

Due to the relatively small number of shots in each round (25), and the well-documented power issues concerning small samples of binary data (Miller and Sanjurjo, 2014), in our primary analysis we: (i) take a similar approach to Koehler and Conley (2003) and measure each statistic across an individual shooter's multiple shooting rounds, and (ii) consider only the shooters who have attempted at least 100 shots, which is the number of shots per shooter in Gilovich et al. (1985)'s controlled test of hot hand shooting. To give a sense of how much streak shooting one might expect to see in 100 shots, a $50 \%$ shooter is expected to generate 12 sequences of three or more hits. The inclusion criterion leads us to consider just 37 of the 123 shooters in our primary analysis. However, these 37 shooters are responsible for the majority (6287 of 9854) of recorded shots in the history of the contest. We will refer to these as the primary shooters. ${ }^{25}$

By comparison, Koehler and Conley (2003) restrict their sample of shooters to those who had at least five shots that immediately followed three or more hits in a row. This is relatively problematic in the sense that: (i) with only five three hit sequences it is mathematically impossible to pass a .05 critical threshold for some of the tests we perform in Section 4, and (ii) it creates an upward bias in the estimate of a shooter's hit rate following 3 or more hits, as this is positively correlated with the number of sequences of three or more hits.

That said, any exclusion criterion that removes shooters that have taken fewer shots in the contest can create a selection towards better shooters. This selection does not bias our statistics themselves in either direction, as they are bench-marked to shooter performance. However, it remains possible that better shooters have either a relatively greater or lesser tendency of getting the hot hand, though we know of no evidence that would indicate either direction to be more plausible. ${ }^{26}$

In any case, in order to test for the sensitivity of our results to the choice of threshold, in Section 4.3 we report robustness checks with 125-shot (5 rounds) and 75-shot (3 round) thresholds, as well as an analysis in which we include all shooters.

\section{Results}

We begin with a basic description of overall shooting performance, then: (i) test for the hot hand (and cold hand) effect in each player, (ii) test whether the number of individual shooters who exhibit hot hand (cold hand) performance is greater than the number expected by chance, (iii) conduct a pooled analysis of the player-clustered average for each of our statistics, and (iv) report robustness checks. In all cases we find strong evidence of hot hand shooting.

\subsection{Summary of overall performance}

The (player-clustered) average shooting percentage among the 37 primary shooters is 55\%, with a minimum of $43 \%$ (Danny Ainge) and a maximum of $69 \%$ (Buddy Hield). By comparison, the lifetime NBA in-game three point percentage among the same shooters is $40 \%$, on average. ${ }^{27}$ The median number of shots among players in the contest is 150 , with a minimum of 100 (10 players) and a maximum of 454 (Craig Hodges).

Regarding possible systematic trends in shooting performance across rounds and contests, shooters' average performance from

\footnotetext{
22 The sampling distribution for $R$ is the only symmetric one.

23 Two-sided test outcomes can be approximated throughout by halving the critical threshold for p-values to .025.

24 The minimum p-value across the realized values of the three test statistics, and across their values in each permutation, are computed with respect to the entire sampling distribution

25 Throughout our analysis we include the two "Dew Zone" shots that were added to each round in 2020, only in considering whether a player is on a streak or not. We exclude the shot outcomes as dependent variables, as they are taken from considerably further away from the basket than all other shots.

26 For example, better shooters might have better and more consistent technique, so be less prone to performance fluctuations such as the hot hand. On the other hand, better shooters might maintain focus better, so be more capable of sustaining long hit streaks.

27 This data was obtained from Basketball-Reference.com's career player statistics https://www.basketball-reference.com/players/ during the 2019-2020 NBA season.
} 
round to round increases by .8 percentage points, which is not statistically significant, and from contest to contest by 1.3 percentage points, which is significant.

Within round, shooters perform signficantly worse on the first three shots, hitting $34 \%$ on the first, $41 \%$ on the second, $49 \%$ on the third, and 57\% thereafter. A similar pattern is observed in controlled shooting studies (Miller and Sanjurjo, 2014) and free throw shooting (Arkes, 2010; Yaari and Eisenmann, 2011).

Outside of the first three shots from each round: (i) a shooter's average performance increases by .2 percentage points with each additional shot, which is not significant, (ii) average hit rates at the five racks are statistically indistinguishable, and (iii) within rack the average hit rates for shots $1-5$ are $50 \%, 59 \%, 59 \%, 61 \%$, and $57 \%$, respectively. In particular, players perform significantly worse than average on racks' first shot, consistent with the analysis of free throw data (Wardrop, 1995; Yaari and Eisenmann, 2011), and significantly better on the fourth. While shooters take significantly more time to shoot the fifth ball on each rack (2.21 s vs. $2.01 \mathrm{~s}$ overall), the two point "money ball," their performance on these shots is not significantly different than the average. ${ }^{28}$

\subsection{Main results}

We test for the prevalence and magnitude of hot hand shooting across shooters, using a variety of statistics. In our primary analysis, we follow the approach of Koehler and Conley (2003), which treats all shots from each shooter as if they were taken under the same conditions. This allows for a more direct comparison of results, and for us to use virtually all of the shot data collected, as we presume most observers of the contest would when forming their hot hand beliefs. We use the term streak throughout to mean a run of three or more consecutive like outcomes, e.g. a streak of hits. Following the primary analysis, in Section 4.3 we report various robustness checks in which we consider alternate streak lengths and account for systematic variations in performance both within and across rounds.

\section{Conditional probability}

One typical conception of the hot hand is that "success breeds success." Accordingly, we explore whether players have a higher hit probability immediately following a streak of hits, as compared to all other recent shot histories. ${ }^{29}$ The difference between these two hit probabilities provides an easy-to-interpret estimate of the magnitude of the hot hand. While this estimate is inherently biased, we adjust for the bias following Miller and Sanjurjo (2018). ${ }^{30}$

In Fig. 1 we report the bias-adjusted estimated hot hand effect for each shooter, along with associated standard error and two-sided 95 percent confidence interval. ${ }^{31}$ of the 37 shooters, 31 shoot better immediately following a streak of hits $(p<.001$, binomial test). Moreover, the magnitude of this improvement is at or above the 95th percentile of the sampling distribution for 8 of these shooters ( $p$ $<.001$, binomial test). ${ }^{32}$ Thus, there is an abnormal prevalence of both directional, and individually significant, hot hand effects.

Players on average shoot an estimated +8.1 percentage points better immediately following a streak of hits $(p<.001$, S.E. $=$ $1.9 \mathrm{pp}){ }^{33}$ This is a large effect size, particularly when taking into account that the larger hot hand effects of a few may be mitigated in the average by a lack of hot hand shooting in others. To put the effect size into context, the difference between the median and top three point shooters in the 2018-2019 season was 10.9 percentage points. ${ }^{34}$ See Table A.1 in Appendix A.1 for individual performance by player name, e.g. Stephen Curry, Larry Bird, and so on.

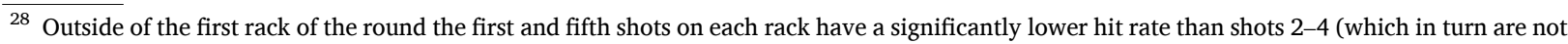
statistically distinguishable from one another). Accordingly, in Section 4.3 we include a robustness check in which the first shot of the rack is placed in one strata, shots two through four in a second strata, and the fifth shot in a third strata.

${ }^{29}$ By contrast, Koehler and Conley (2003) compare the probability of hit immediately following a streak of hits with that following a streak of misses of the same length. Our approach uses relatively more data, and makes it more likely that a positive effect is driven by superior performance on hit streaks rather than inferior performance on miss streaks (see Miller and Sanjurjo (2014) for a discussion).

${ }^{30}$ We approximate the bias for each player by simulating shot outcomes based on his observed shooting percentage.

31 Though hot hand tests are one-sided (see Section 3) we show the more familiar two-sided confidence intervals in Fig. 1. The confidence interval for any individual player should be interpreted with caution, as it is not corrected for multiple comparisons.

32 By contrast just 1 of the 37 shooters has a significant "anti-hot hand" effect, i.e. he shoots significantly worse immediately following a streak of hits.

${ }^{33}$ Before adjusting for the bias the estimate is 5.7 percentage points. This is the unweighted average across players. See Appendix A.3 for an estimate that instead places more weight on the shooters that generate more observations. The standard error is computed based on the assumption of independence across all trials, and normality. In particular, defining player $i$ 's difference $\widehat{D}_{k}^{i}:=\widehat{P}^{i}$ (hit $\mid k$ hits $)-\widehat{P}^{i}($ hit $\mid \neg k$ hits), the variance satisfies $\widehat{\operatorname{Var}}\left(\widehat{D}_{k}^{i}\right)=\widehat{\operatorname{Var}}\left(\widehat{P}^{i}(\right.$ hit $\mid k$ hits $\left.)\right)+\widehat{\operatorname{Var}}\left(\widehat{P}^{i}(\right.$ hit $\mid \neg k$ hits $\left.)\right)$ for each player $i$. Simulations reveal that the associated $(1-\alpha) \times 100 \%$ confidence intervals with radius $z_{\alpha / 2} \times \widehat{\operatorname{Var}}\left(\bar{D}_{k}\right)^{1 / 2}$ (where the mean difference is given by $\bar{D}_{k}:=(1 / n) \sum_{i=1}^{n} \widehat{D}_{k}^{i}$ ) have the appropriate coverage-i.e. $(1-\alpha$ /2) $\times 100 \%$ of the time the true difference is greater than $\bar{D}_{k}-z_{\alpha / 2} \times \widehat{\operatorname{Var}}\left(\bar{D}_{k}\right)^{1 / 2}$, for both Bernoulli trials and the positive feedback models of the hot hand.

34 This data was obtained from ESPN's “NBA Player Stats 2018-2019” https://www.espn.com/nba/stats/player on May 12, 2020.
} 


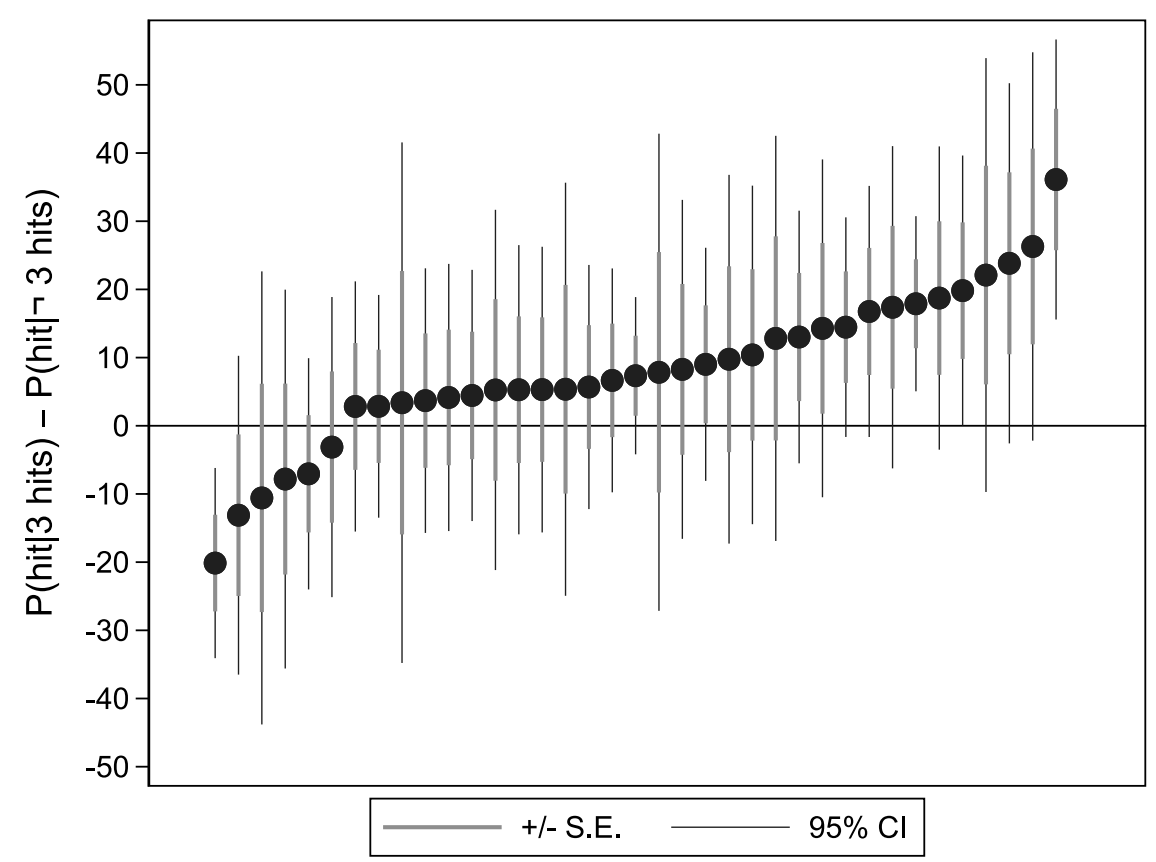

Fig. 1. Estimated (bias-adjusted) hot hand effect for each shooter, with associated standard error and two-sided 95 percent confidence interval.

While we find strong evidence of shooters performing relatively better when on a streak of hits, we find no similar evidence of shooters performing relatively worse when on a streak of misses (see Appendix A.2).

Streak frequency, momentum, and length

In order to get a broader view of hot hand performance across the 37 shooters, Fig. 2 plots each shooter's hit streak statistics for frequency (percentage of shots that immediately follow a streak of hits), momentum (hit rate when on a streak of hits), and length (length of longest run of hits), as well as his runs statistic (number of runs of hits plus number of runs of misses). Each of these four statistics is depicted in a separate cell in the figure. In each cell, each shooter's corresponding statistic, averaged across rounds, is plotted against its median under the null (based on the permutation distribution). If the shooter's statistic is on the side of the median (gray line) predicted by the hot hand hypothesis, we refer to this as the "hot side" of the median; for the three hit streak statistics, $H_{F}$, $H_{M}$, and $H_{L}$, the hot side is above the median, while for the runs statistic, $R$, it is below.

We test for whether there are more directional hot hand effects than would be expected by chance, for each of these four statistics. Fig. 2 shows that the hit streak frequency $\left(H_{F}\right)$ statistic is on the hot side of the median for 27 of the 37 shooters; we would observe this many directionally hot shooters, or more, in roughly four out of every one thousand studies if the null hypothesis that each player shoots with a constant probability of success were true ( $p=.004$, binomial test). Similarly, the hit streak momentum $H_{M}$ statistic is on the hot side of the median for 32 of the 37 shooters $(p<.001)$, the hit streak length $H_{L}$ for 23 of the 37 ( $\left.p=.094\right)$, and the runs $R$ statistic for 29 of the $37(p<.001) .{ }^{35}$

We also test whether there are more individually significant hot hand effects than would be expected by chance. The hit streak frequency $\left(H_{F}\right)$ statistic is at or above the 95th percentile of the null distribution for 7 of the 37 shooters $(p=.002$, binomial test), the hit streak momentum $H_{M}$ statistic for 3 of the 37 shooters $(p=.282)$, the hit streak length $H_{L}$ for 7 of the $37(p=.002)$, and the runs $R$ statistic for 8 of the $37(p<.001) .^{36}$

Thus, under the null hypothesis that each player shoots with a constant probability of success, we would almost never observe as many shooters with directional hot hands as what we observe here, nor would we observe as many shooters with individually significant hot hands. In Appendix A. 2 we show that the analogous miss streak statistics do not indicate a comparable tendency towards directional, nor individually significant, cold hands.

\footnotetext{
35 We address the issue of multiple hypotheses with the minimum p-value across $H_{F}, H_{M}$, and $H_{L}$ (see Section 3 ), which is below the median of the corresponding minimum $\mathrm{p}$-value sampling distribution for 28 of the 37 shooters $(p=.001)$.

${ }^{36}$ Similarly, the minimum p-value across $H_{F}, H_{M}$, and $H_{L}$ is at or below the fifth percentile of the corresponding minimum p-value sampling distribution for 7 of the 37 shooters $(p=.002)$.
} 

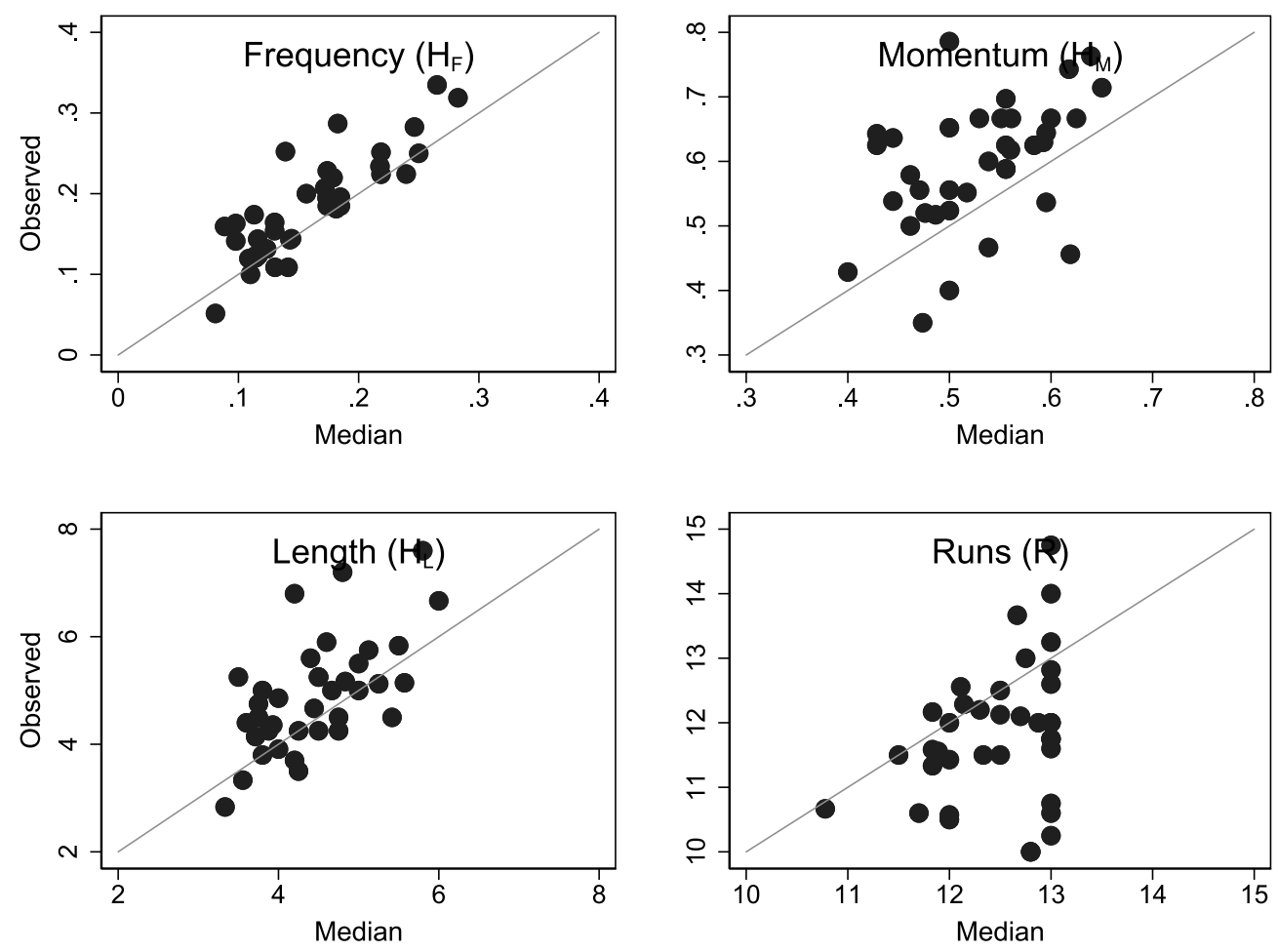

Fig. 2. Hit streak and runs statistics for each shooter, with respect to his median under the null.

A pooled analysis of the data yields similar results. In particular, in Table 1 we report the hit streak and runs statistics, averaged across all shooters. ${ }^{37}$ Each statistic is reported in standard deviations from the mean of its null distribution, with associated p-values in parentheses. For example, the value of .88 for $H_{F}$ indicates that shooters, on average, find themselves on a hit streak more frequently than would be expected under the null, and that the difference corresponds to nearly a full standard deviation increase in performance. All three of the hit streak statistics, as well as the runs statistic, are highly significant. A similar pattern does not emerge for the miss streak statistics (see Appendix A.2).

\section{Table 1}

Average hit streak and runs statistics across players, in terms of standard deviations from the mean, computed from each player's sampling distribution, with p-values in parentheses.

\begin{tabular}{lc}
\hline Statistic & Std. Dev. from Mean \\
\hline Hit Streak Frequency $\left(H_{F}\right)$ & 0.88 \\
& $(<.001)$ \\
Hit Streak Momentum $\left(H_{M}\right)$ & 0.63 \\
& $(<.001)$ \\
Hit Streak Length $\left(H_{L}\right)$ & 0.74 \\
& $(<.001)$ \\
Total Runs $(R)$ & -0.69 \\
& $(<.001)$ \\
\hline
\end{tabular}

\footnotetext{
$\overline{37}$ The streak length and the runs statistics are averaged across rounds for each player.
} 


\subsection{Robustness checks}

To test how sensitive the results reported in Section 4.2 are to specific features of our primary analysis, we consider multiple alternative: (i) streak lengths, (ii) permutation strata and fixed effects, and (iii) shooter inclusion criteria. In addition, we address the "warm-up" effect on the first three shots from each round. The results summarized here can be seen in greater detail in Appendix A.3.

\section{Streak length}

Considering streak lengths of two, and four, rather than three, binomial tests continue to reveal an abnormal prevalence of directional hot hand shooters, and an abnormal prevalence of individually significant hot hand shooters. ${ }^{38}$ Shooting performance when on a streak of hits, as compared to any other recent shot history, decreases from +8.1 percentage points in our primary analysis to $+6.6 \mathrm{pp}(p<.001$, S.E. $=1.6 \mathrm{pp})$ and $+6.7 \mathrm{pp}(p=.006$, S.E. $=2.7 \mathrm{pp})$, respectively.

\section{Permutation strata}

We permute shot outcomes not only on the shooter level, but also on the following more granular levels: (i) shooter $\times$ contest $\times$ round, ${ }^{39}$ (ii) shooter $\times$ contest $\times$ round $\times$ shot number on rack, ${ }^{40}$ and (iii) shooter $\times$ contest $\times$ round $\times$ shot number on rack with a separate strata for the first three shots in the round. Notice that these alternative stratification schemes affect the sampling distribution of each statistic, but not its raw effect size. As the number of strata increase, the average standardized hit streak and runs statistics decrease slightly in size, but remain highly significant in the pooled test. In most cases, binomial tests continue to reveal an abnormal prevalence of directional hot hand shooters, and an abnormal prevalence of individually significant hot hand shooters. Thus, despite these alternative stratifications eliminating, for example, the possibility of detecting forms of hot hand shooting that activate between (rather than within) rounds, and contests, the hot hand effect persists.

\section{Shooter inclusion criteria}

Instead of including all shooters that have taken at least 100 shots, we vary this threshold to 125, 75, and 1 (to include all shooters). Under all thresholds binomial tests continue to reveal an abnormal prevalence of hot hand shooters, and an abnormal prevalence of individually significant hot hand shooters. ${ }^{41}$ The average increase in performance when shooters are on a streak of three or more hits (as compared to any other recent shot history) for the 125 shot threshold is $+8.0 p p(p<.001$, S.E. $=2.1 \mathrm{pp}$ ), for the 75 shot threshold is $+6.5 \mathrm{pp}(p<.001$, S.E. $=2.0 \mathrm{pp})$, and for all shooters with at least two instances of a shot following three or more hits is $+9.0 \mathrm{pp}(p$ $<.001$, S.E. $=2.3$ pp). ${ }^{42}$

\section{Warm-up effect}

In Section 4.1 we report that players, on average, shoot systematically worse on the first three shots of the round. While the validity of discarding these shots is debatable, given that they may affect observers' hot hand beliefs, if we exclude these shots from the primary analysis, in most cases binomial tests continue to reveal an abnormal prevalence of directional hot hand shooters, or an abnormal prevalence of individually significant hot hand shooters. The increase in shooter performance is $+5.7(p=.002$, S.E. $=2.0 \mathrm{pp})$ percentage points when on a streak of three or more hits (as opposed to any other recent shot history), as compared to $+8.1 \mathrm{pp}$ when the first three shots of the round are included.

\section{Sensitivity of estimates to varying multiple features simultaneously}

So far we have explored the robustness of our primary analysis to varying one feature of the analysis at a time. Now, we explore the sensitivity of the estimated improvement in shooting performance when on a streak of hits to varying multiple features of the analysis simultaneously.

In particular, we consider multiple specifications of a linear probability model with shooter fixed effects, in which we incrementally interact additional fixed effects for: (i) contest $\times$ round ("session"), (iii) three rack locations: corner, wing, and center ("rack"), and (iv) order of the shot within the rack ("ball"). These different specifications allow us to observe the impact of incremental controls on the size of the improvement in shooting performance, whereas varying permutation strata (as we did above) does not. ${ }^{43}$

In addition, we cross these alternative fixed effect specifications with the four separate shooter inclusion criteria considered above $(125,100,75$, or 1 shots), and either drop or include the first three shots in the round to account for the observed warm-up effect. In total, this yields 64 alternative specifications of the model.

\footnotetext{
38 The frequency $H_{F}$ and momentum $H_{M}$ hit streak statistics remain similar in size, and highly significant in the pooled test. Runs $R$ and longest run $H_{L}$ are unaffected by altering the defined streak length.

39 If a shooter participates in two contests of two rounds each, i.e. four sessions total, then the "contest $\times$ round" level strata means that shots are permuted separately within each of the shooter's four sessions, keeping the number of hit shots constant within each session.

40 The first shot of the rack is placed in one strata, shots two through four in a second strata, and the fifth shot in a third strata.

41 The average hit streak and runs statistics decrease in size as more low volume shooters are included in the analysis (by up to as much as around 50 percent), but remain highly significant in the pooled test.

42 At least two observations are necessary to compute standard errors for individual shooters. If we instead follow Koehler and Conley (2003), by only including all shooters that have at least five such instances, then the effect is $+8.9 \mathrm{pp}(p<.001$, S.E. $=2.0 \mathrm{pp})$.

${ }^{43}$ Varying the permutation strata changes the sampling distributions but not the estimated effect sizes.
} 
We observe that the average estimated improvement in shooting performance immediately following a streak of hits is significantly different (and larger) than zero at the 5 percent level for 52 of the 64 specifications, and at the 10 percent level for 60 of the 64 . The minimum estimate is $+2.7 \mathrm{pp}(p=.118$, S.E. $=1.7 \mathrm{pp})$ and the maximum estimate is $+9.9 \mathrm{pp}(p<.001$, S.E. $=2.8 \mathrm{pp})$. The largest estimates, in general, correspond to specifications with more fixed effects and that include all shots from the round.

For more details, including all 64 estimated effect sizes represented in a single specification curve (Simonsohn et al., 2019), see Fig. A.7 in Appendix A.3. In addition, we perform a similar analysis to test whether shooters "heat up" within racks. We find that they do not. See Fig. A.8 in Appendix A.3.

\section{Discussion}

The annual NBA Three Point Contest has been described as an ideal setting to study the hot hand, given that its elite professional shooters perform under conditions that approximate those of in-game NBA shooting, but in the absence of many of the confounds present there. In contrast with the results of Koehler and Conley (2003)_a prominent replication of the original hot hand fallacy study of Gilovich et al. (1985)—we find considerable evidence of hot hand shooting.

The abnormal prevalence of significant hot hand shooters that we observe translates to roughly one of every five players on a team exhibiting a hot hand that is large enough, or frequent enough, to be detectable. Further, despite considerable variation in the size (and sometimes even the sign) of the hot hand effect across shooters, the average shooter experiences an increase in performance of roughly +5 to +9 percentage points when on a streak of three or more hits. The magnitude of this average effect size is striking, given that it is not clear that expert players and coaches (or even the average fan) tend to believe in more than merely "sometimes some players experience a hot hand." 44

Moreover, it is important to note that the average increase in shooting performance that we report represents a conservative estimate of the magnitude of the true hot hand effect. The reason why is that hitting three shots in a row is only a noisy signal of a shooter's underlying performance state, which results in an attenuation bias in the estimated effect size (Stone, 2012; Arkes, 2013; Miller and Sanjurjo, 2018). ${ }^{45}$

When combining the results reported here on elite NBA shooters, with those reported on professional, semi-professional, and collegiate-level shooters in all extant controlled shooting experiments (Miller and Sanjurjo, 2014), the conservative measures of shooters' average improvement in performance when on streak of hits, across environments, ranges from +5 to +9 percentage points and up (Miller and Sanjurjo, 2018; 2014). ${ }^{46}$ As mentioned in Section 4, these effect sizes are substantial in NBA shooting terms.

Thus, despite the hot hand fallacy being generally recognized as a "massive and widespread cognitive illusion" for the better part of the last three and a half decades (Kahneman, 2011), with the results presented here, the current body of evidence strongly suggests that it is not a fallacy to believe in the hot hand-whether the belief be directed towards college, semi-professional, or the world's best professional shooters. $^{47}$

It is our hope that laying the extreme hot hand fallacy notion to rest might help direct more attention to the subtle, but important, calibration questions that remain unanswered. An example of such a question is whether-and to what extent-people systematically over (or under) estimate the prevalence of hot hand shooting, or the magnitude of the hot hand when it occurs-what might be referred to as hot hand bias, rather than fallacy.

\section{Declaration of Competing Interest}

We have no conflicts of interest.

\footnotetext{
$\overline{44}$ Gilovich et al. (1985) report the following feedback regarding hot hand beliefs, after interviewing eight players from an NBA team: "Most of the players (six out of eight) reported that they have on occasion felt that after having made a few shots in a row they 'know' they are going to make their next shot- that they 'almost can't miss.' Five players believed that a player 'has a better chance of making a shot after having just made his last two or three shots than he does after having just missed his last two or three shots.' (Two players did not endorse this statement and one did not answer the question.)...”. Similarly, in a survey of semi-professional teammates (Miller and Sanjurjo, 2014) only one of eight players believed that all eight of them shot better after having hit several in a row.

45 This bias is illustrated for a variety of models of hot hand shooting in Miller and Sanjurjo (2014).

${ }^{46}$ In Footnote 7 we mention some of the challenges to testing for the hot hand in game data. Nevertheless, while Gilovich et al. (1985) find no evidence of hot hand shooting in their analyses of NBA live ball and free throw shooting data, more recent studies, that are more powered and add far more control, find modest increases in players' shooting percentages following recent shooting success (Arkes, 2010; Yaari and Eisenmann, 2011; Bocskocsky et al., 2014).

${ }^{47}$ While we observe little evidence of cold hand shooting here, considerable evidence of it has been observed in multiple controlled shooting experiments particularly in those with non-professional shooters (Miller and Sanjurjo, 2014). Interestingly, the data from an experiment with professional shooters studied there reveals relatively less cold hand shooting. When combined with the results presented here, this suggests that professional players may be relatively more skilled at extending hot streaks and ending cold streaks.
} 


\section{Appendix A}

\section{A1. Table with individual shooter performance}

Table A. 1 complements the results presented in Section 4.2, particularly those of Fig. 1. It reports each shooter's name, number of shots, overall hit rate, and raw relative increase in shooting percentage when on a streak of hits (as compared to any other recent shot history), along with the respective bias-adjusted increase. The highest bias-adjusted increase across shooters is $+36 \mathrm{pp}$ (Hubert Davis), the lowest is -20pp (Peja Stojakovic), and the average is $+8 \mathrm{pp}$ (as reported in Section 4.2).

\section{Table A.1}

Each shooter's total number of shots, overall shooting percentage, and increase in shooting percentage immediately following a streak of three or more hits in a row (as compared to any other recent shot history) first raw then bias-adjusted.

\begin{tabular}{|c|c|c|c|c|}
\hline \multirow[b]{2}{*}{ Player } & \multirow[b]{2}{*}{ \# shots } & \multirow[b]{2}{*}{ Hit rate } & \multicolumn{2}{|c|}{$\begin{array}{l}\text { Change in hit rate after } \\
\text { hitting } 3 \text { or more }\end{array}$} \\
\hline & & & Raw & Bias-adjusted \\
\hline Davis, Hubert & 125 & .53 & .33 & .36 \\
\hline Richardson, Quintin & 100 & .46 & .21 & .26 \\
\hline Lewis, Rashard & 123 & .46 & .20 & .24 \\
\hline Durant, Kevin & 100 & .48 & .18 & .22 \\
\hline Scott, Dennis & 125 & .57 & .18 & .20 \\
\hline Hornacek, Jeff & 160 & .51 & .16 & .19 \\
\hline Curry, Stephen & 250 & .64 & .17 & .18 \\
\hline Korver, Kyle & 125 & .54 & .15 & .17 \\
\hline Legler, Tim & 150 & .63 & .15 & .17 \\
\hline Allen, Ray & 247 & .56 & .13 & .15 \\
\hline Pierce, Paul & 125 & .49 & .11 & .14 \\
\hline Bird, Larry & 211 & .57 & .12 & .13 \\
\hline Billups, Chauncey & 100 & .47 & .08 & .13 \\
\hline Porter, Terry & 160 & .49 & .08 & .10 \\
\hline Belinelli, Marco & 100 & .57 & .07 & .10 \\
\hline Kapono, Jason & 150 & .66 & .08 & .09 \\
\hline Cook, Daequan & 100 & .56 & .05 & .08 \\
\hline Beal, Bradley & 100 & .52 & .04 & .08 \\
\hline Hodges, Craig & 454 & .56 & .07 & .07 \\
\hline Price, Mark & 199 & .60 & .05 & .07 \\
\hline Thompson, Klay & 174 & .63 & .04 & .06 \\
\hline Schrempf, Detlef & 124 & .48 & .02 & .05 \\
\hline Person, Wesley & 136 & .60 & .03 & .05 \\
\hline Rice, Glen & 197 & .49 & .03 & .05 \\
\hline Jones, James & 100 & .57 & .02 & .05 \\
\hline Kerr, Steve & 208 & .56 & .03 & .04 \\
\hline Miller, Reggie & 235 & .53 & .03 & .04 \\
\hline Novitski, Dirk & 273 & .53 & .03 & .04 \\
\hline Ainge, Danny & 148 & .43 & .00 & .03 \\
\hline Ellis, Dale & 320 & .51 & .02 & .03 \\
\hline Booker, Devin & 194 & .61 & .02 & .03 \\
\hline Hield, Buddy & 104 & .66 & -.05 & -.03 \\
\hline Irving, Kyrie & 199 & .60 & -.08 & -.07 \\
\hline Arenas, Gilbert & 100 & .56 & -.11 & -.08 \\
\hline Lenard, Voshon & 100 & .53 & -.14 & -.11 \\
\hline Barros, Dana & 184 & .49 & -.16 & -.13 \\
\hline Stojakovic, Peja & 287 & .62 & -.21 & -.20 \\
\hline Average & 170 & .55 & .06 & .08 \\
\hline
\end{tabular}




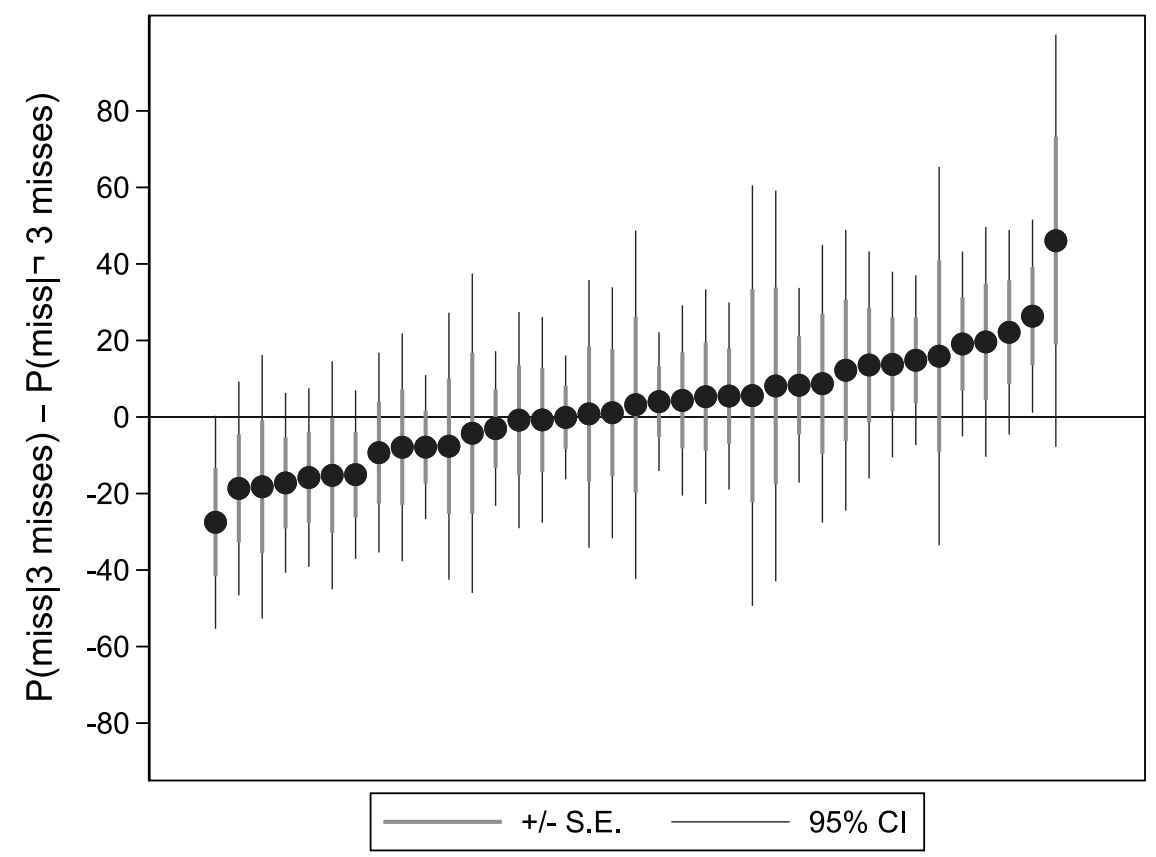

Fig. A.1. Estimated (bias-adjusted) cold hand effect for each shooter, with associated standard error and 95 percent confidence interval.
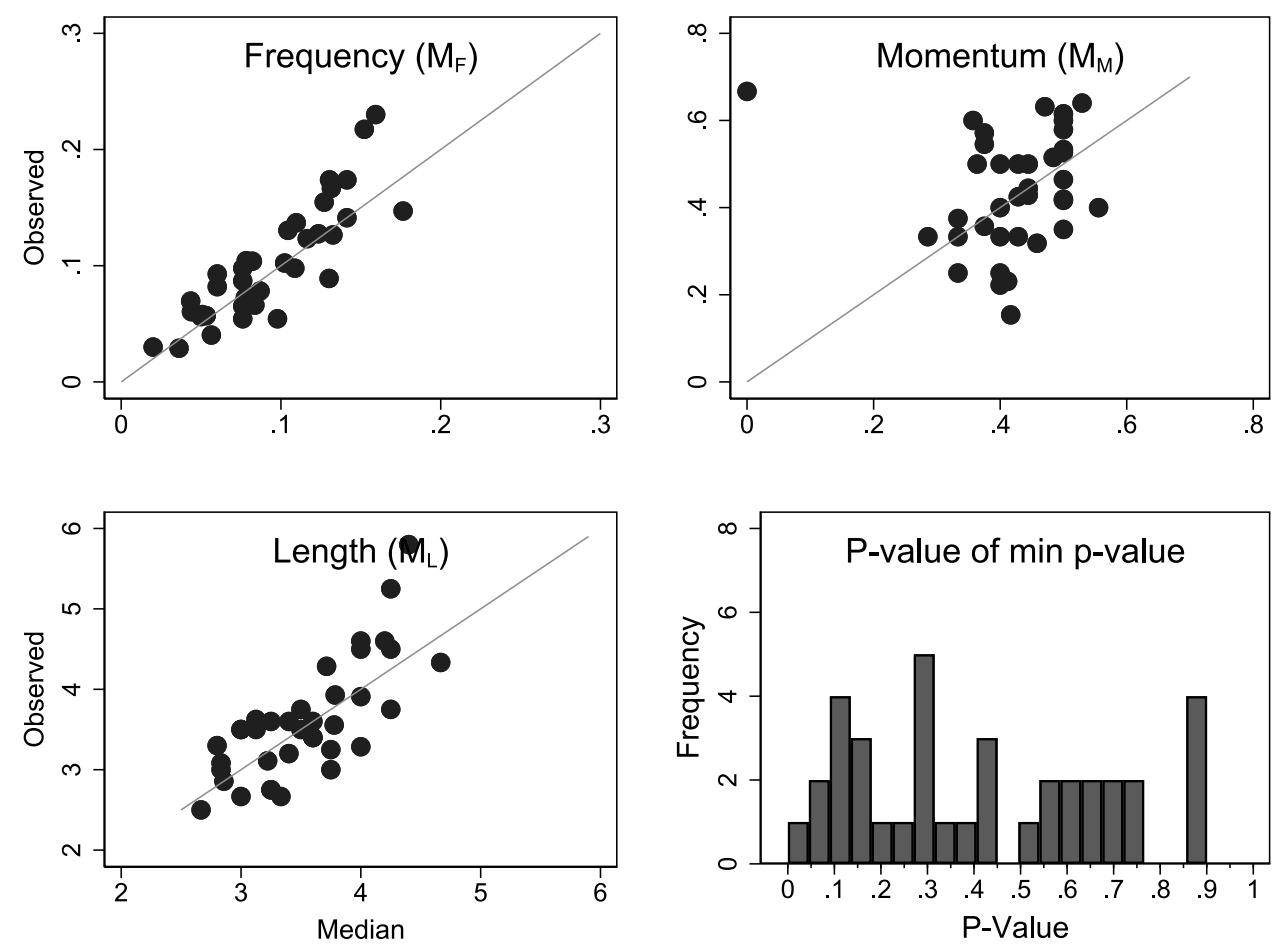

Fig. A.2. Miss streak statistics for each shooter, with respect to his median under the null, and histogram aggregating the p-value of each shooter for his minimum p-value across the three miss streak statistics.

\section{A2. Testing for the cold hand}

We first present an analysis on the cold hand that is analogous to the hot hand analysis performed in Section 4.2, on the 37 primary shooters. Then we present robustness checks in which we vary streak length, permutation strata, and shooter inclusion criteria, and compare hot hand and cold hand results side-by-side to facilitate comparison. 
Table A.2

Average hit and miss streak statistics across players, in terms of standard deviations from the mean, computed from each player's sampling distribution, with p-values in parentheses.

\begin{tabular}{lc}
\hline Statistic & Std. Dev. from Mean \\
\hline Hit Streak Frequency $\left(H_{F}\right)$ & .88 \\
& $(<.001)$ \\
Hit Streak Momentum $\left(H_{M}\right)$ & .63 \\
& $(<.001)$ \\
Hit Streak Length $\left(H_{L}\right)$ & .74 \\
& $(<.001)$ \\
Total Runs $(R)$ & -.69 \\
& $(<.001)$ \\
Miss Streak Frequency $\left(M_{F}\right)$ & .32 \\
& $(.027)$ \\
Miss Streak Momentum $\left(M_{M}\right)$ & .08 \\
& $(.317)$ \\
Miss Streak Length $\left(M_{L}\right)$ & .11 \\
& $(.257)$ \\
\hline
\end{tabular}
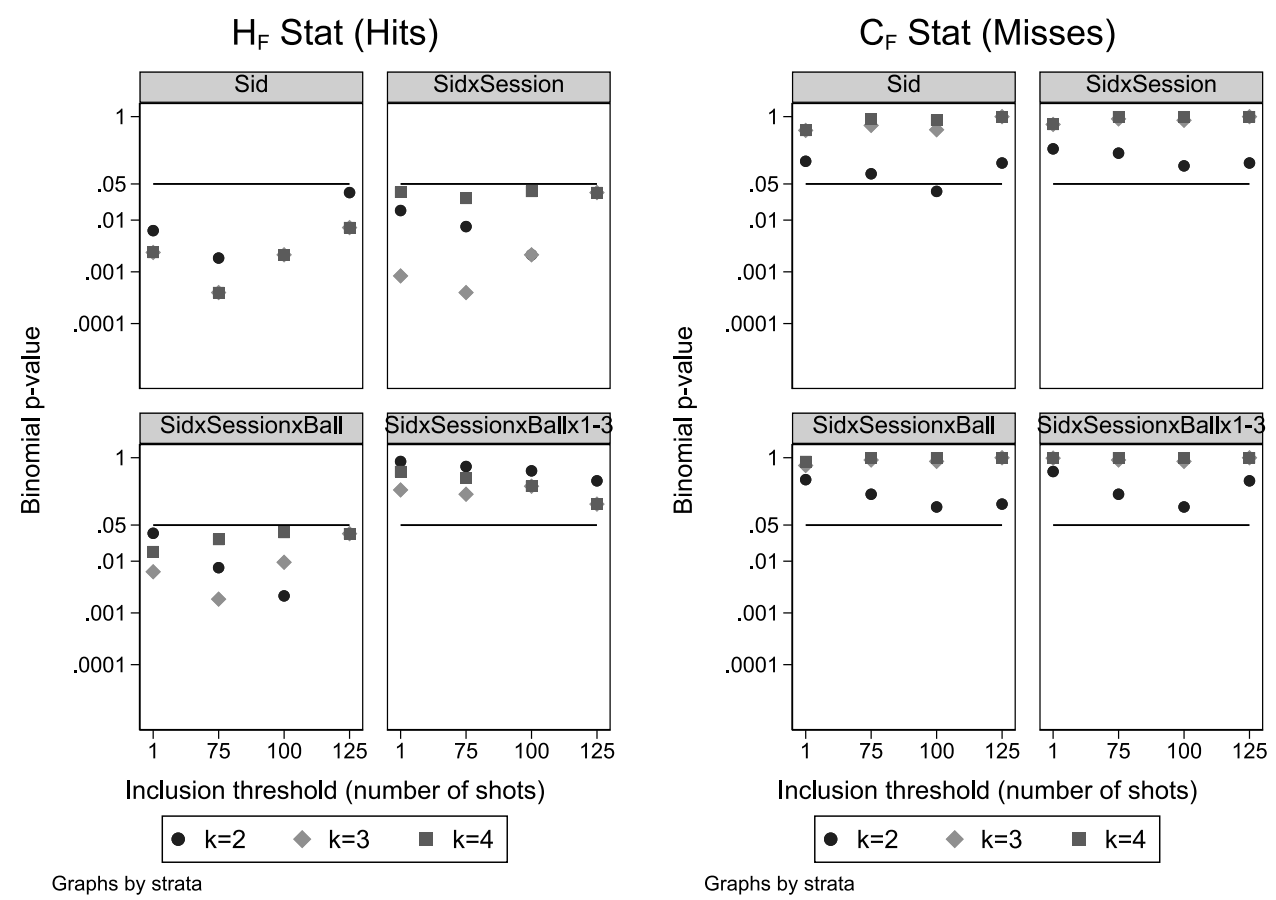

Fig. A.3. P-values of binomial tests for frequency statistics in hits $H_{F}$ (left panel) and misses $M_{F}$ (right panel), for various streak lengths ( $k$ ), restrictions on permutations (each represented in a different cell), and shooter inclusion criteria (on horizontal axis). Restrictions on permutations increase incrementally by interacting strata on shooter (Sid), round $x$ contest (Session), ball number on rack (Ball), and "warm-up" shots (1-3) of the round. As the shooter inclusion criterion on shots changes from 1 to 75,100 , and 125, binomial tests are based on a decreasing number of shooters, e.g. for $k=3$ the number decreases from 118 to 46,37 , and 25 , respectively.

\section{Cold hand in primary shooters}

As explained in Section 3, repeating our analysis of hit streaks for miss streaks allows us to separate the hot hand from the cold hand in streak shooting. Here we repeat the analysis from Section 4.2, but for cold hand, rather than hot hand, shooting.

We first explore whether players have a lower hit probability immediately following a streak of misses, as compared to all other recent shot histories. In particular, In Fig. A.1 we report the (bias-adjusted) estimate of the cold hand effect for each shooter, along with 

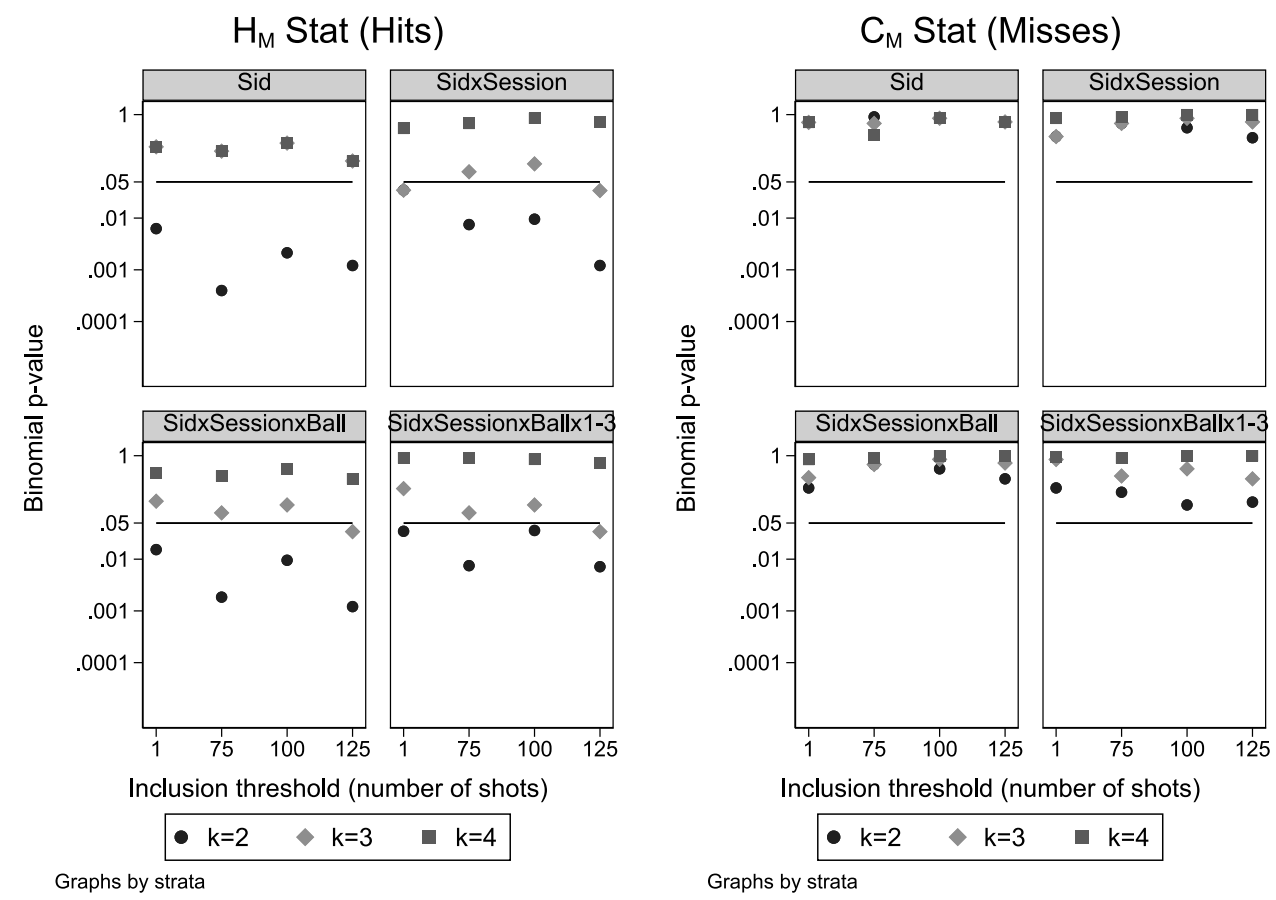

Fig. A.4. P-values of binomial tests for momentum statistics in hits $H_{M}$ (left panel) and misses $M_{M}$ (right panel), for various streak lengths ( $k$ ), restrictions on permutations (each represented in a different cell), and shooter inclusion criteria (on horizontal axis). Restrictions on permutations increase incrementally by interacting strata on shooter (Sid), round $x$ contest (Session), ball number on rack (Ball), and "warm-up" shots (1-3) of the round. As the shooter inclusion criterion on shots changes from 1 to 75, 100, and 125, binomial tests are based on a decreasing number of shooters, e.g. for $k=3$ the number decreases from 118 to 46,37 , and 25 , respectively.

associated standard error and 95 percent confidence interval. ${ }^{48}$ Of the 37 shooters, just 21 shoot relatively worse following a streak of misses ( $p=.256$, binomial test). Further, just 2 out of 37 players ( $p=.558$, binomial test) produce a cold hand effect that is significant at the individual level (i.e. where $p<.05$, $t$-test).

Players on average shoot an estimated -2.4 percentage points worse immediately following a streak of three or more misses, as compared to their performance immediately following any other recent shot history $(p=.18$, S.E. $=2.7 \mathrm{pp}){ }^{49}$

We test for whether there are more directional cold hand effects than would be expected by chance, for the three miss streak statistics, and the minimum p-value across the three miss streak statistics of each shooter. Fig. A.2 shows that the frequency $M_{F}$, momentum $M_{M}$, and length $M_{L}$ statistics are on the cold side of the median for 22 ( $p=.162$, binomial test), 18 ( $\left.p=.629\right)$, and 18 ( $p=$ .629) of the 37 primary shooters, respectively. They are on the cold side of the 95th percentile for $2(p=.558), 1(p=.850)$, and $1(p=$ .850) of the 37 shooters, respectively. Similarly, the minimum $p$-value across $M_{F}, M_{M}$, and $M_{L}$ is below the median of the corresponding minimum p-value sampling distribution for 22 of the 37 shooters $(p=.162)$, and below the 5 th percentile for 2 of the $37(p=.558)$. This, combined with the analogous results for hit streak (and runs) statistics reported in Section 4.2, clearly indicate that most of the abnormal streak shooting detected by the runs statistic there is driven by the hot hand and not the cold hand.

Table A. 2 reports the results of a pooled analysis of the miss streak statistics. The standard deviations from the mean are .32 for the miss streak frequency $M_{F}$ statistic ( $\left.p=.027\right)$, .08 for the miss streak momentum $M_{M}$ statistic $(p=.317$ ), and .11 for the miss strength length $M_{L}$ statistic ( $\left.p=.257\right)$. One can see in the table that the average effect sizes are considerably smaller for miss streak statistics, as compared to the average hit streak statistics reported in Section 4.2. In addition, only $\boldsymbol{M}_{F}$ is statistically significant in misses, whereas all three hit streak statistics are highly statistically significant. This again indicates that most of the streakiness detected by the runs statistic is due to the hot hand, and not the cold hand.

\footnotetext{
48 The confidence interval for any individual player should be interpreted with caution, as it is not corrected for multiple comparisons.

49 Before adjusting for the bias players shoot +1.4 percentage points better following a streak of misses.
} 
Cold vs. hot hand robustness checks

We extend the binomial tests of cold hand shooting presented above to: (i) alternative streak lengths of two, three, and four, (ii) permutations stratified on the level of: shooter ("Sid"), shooter $\times$ contest $\times$ round ("Sid $\times$ Session"), shooter $\times$ contest $\times$ round $\times$ shot (ball) on rack ("Sid $\times$ Session $\times$ Ball"), and shooter $\times$ contest $\times$ round $\times$ ball on rack and a separate strata for the first three shots of the round ("Sid $\times$ Session $\times$ Ball $\times 1-3$ "), and (iii) include all shooters that have taken at least 125, 100, 75, or 1 shot(s).

We present a separate figure for each of the frequency $M_{F}$ (Fig. A.3), momentum $M_{M}$ (Fig. A.4), and length $M_{L}$ (Fig. A.5) statistics, as well as the p-value of the shooters' minimum p-value across the three miss streak statistics (Fig. A.6). In the right panel of each figure we report the associated p-values for the miss streak statistic, and to facilitate comparison, in the left panel of each figure we report the corresponding results for the analogous hit streak statistic. The vertical axis of each figure indicates the binomial p-value of the test, logarithmically scaled for $p$-values of .0001 to .01.

In each of the four figures one can see that the p-values of binomial tests are considerably lower for hit streak statistics than for miss streak statistics. In particular, test results for hit streak statistics tend to be highly significant across the various conditions considered, whereas results for miss streak statistics do not.
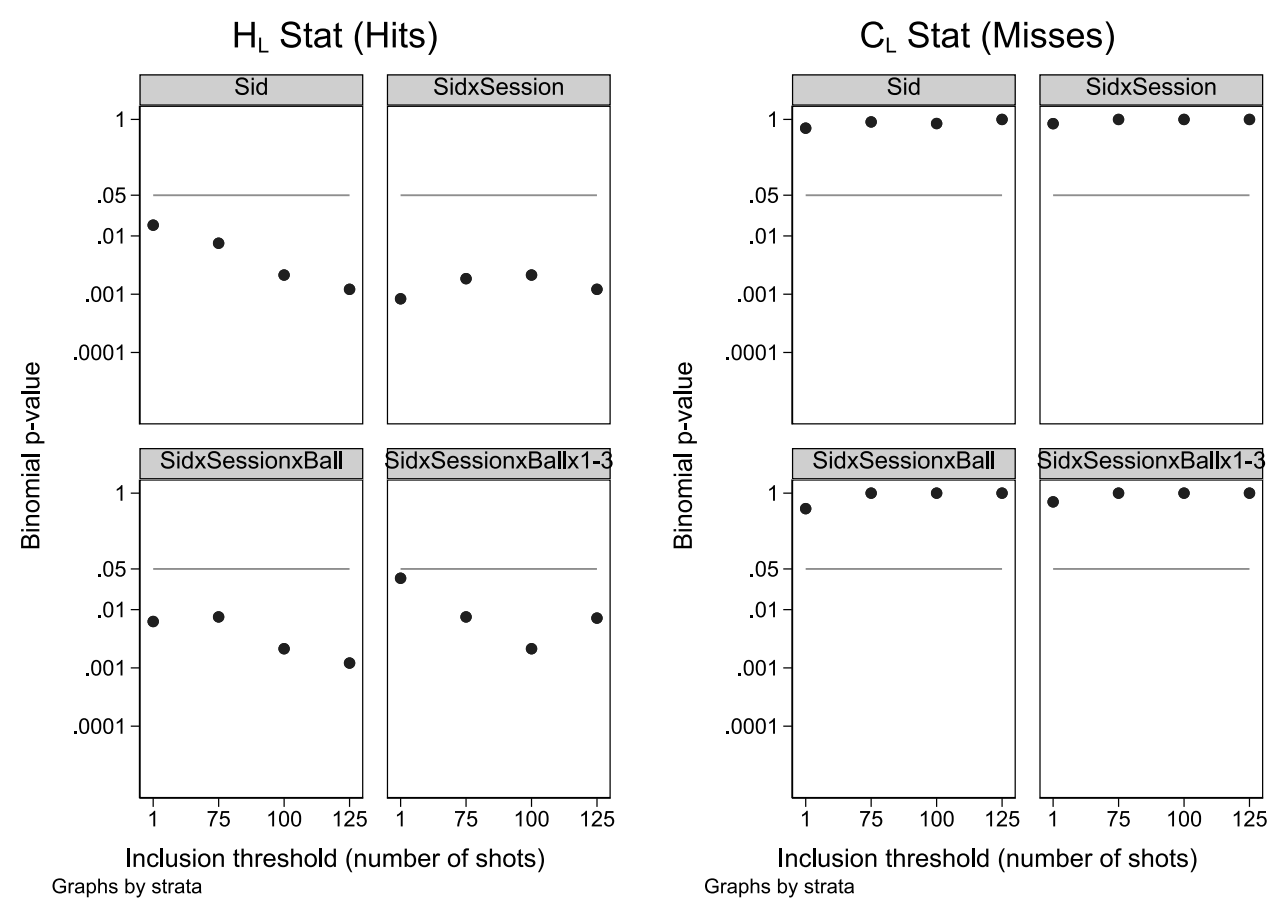

Fig. A.5. P-values of binomial tests for length statistics in hits $H_{L}$ (left panel) and misses $M_{L}$ (right panel), for various streak lengths ( $k$ ), restrictions on permutations (each represented in a different cell), and shooter inclusion criteria (on horizontal axis). Restrictions on permutations increase incrementally by interacting strata on shooter (Sid), round $x$ contest (Session), ball number on rack (Ball), and "warm-up" shots (1-3) of the round. As the shooter inclusion criterion on shots changes from 1 to 75,100 , and 125, the number of shooters that binomial tests are based on decreases from 123 to 46, 37, and 25, respectively. 

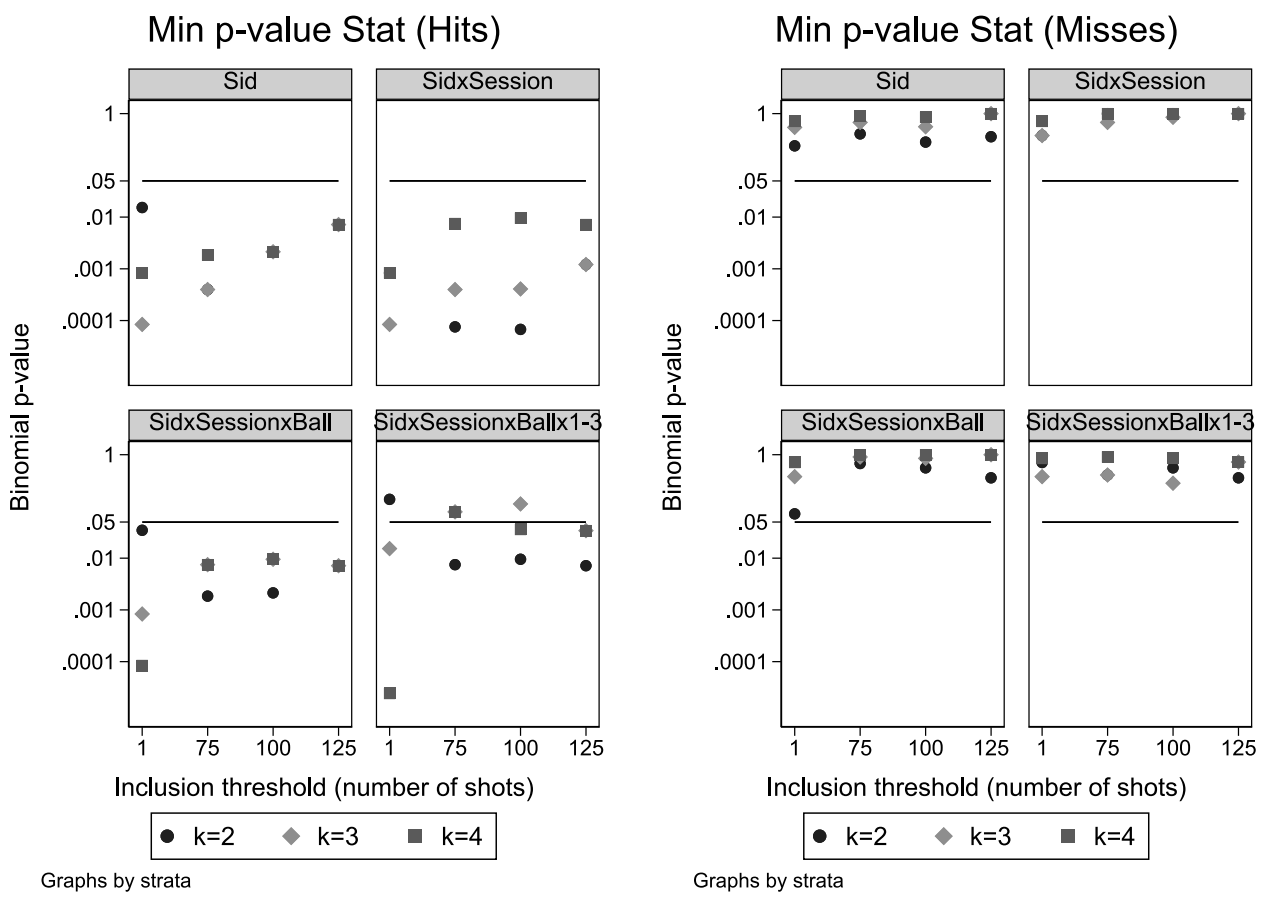

Fig. A.6. P-values of binomial tests for shooter minimum p-value across the three hit streak statistics $H_{F}, H_{M}$, and $H_{L}$ (left panel), and three miss streak statistics $M_{F}, M_{M}$, and $M_{L}$ (right panel), for various streak lengths ( $k$ ), restrictions on permutations (each represented in a different cell), and shooter inclusion criteria (on horizontal axis). Restrictions on permutations increase incrementally by interacting strata on shooter (Sid), round $x$ contest (Session), ball number on rack (Ball), and "warm-up" shots (1-3) of the round. As the shooter inclusion criterion on shots changes from 1 to 75,100 , and 125, binomial tests are based on a decreasing number of shooters, e.g. for $k=3$ the number decreases from 118 to 46 , 37 , and 25 , respectively.

\section{A3. Robustness checks on hot hand performance}

We report the robustness checks summarized in Section 4.3, in detail, and interpret results.

\section{Streak length}

While there is no clear consensus regarding how many consecutive outcomes constitute a streak, in our primary analysis we choose three as the critical number, as it: (i) is commonly used in previous studies (Rao, 2009b; Koehler and Conley, 2003; Gilovich et al., 1985; Avugos et al., 2013), (ii) coincides with the threshold that human observers use to categorize consecutive outcomes as a streak (Carlson and Shu, 2007), and (iii) is a reasonable middle-ground given the following trade-off: while higher streak thresholds provide a stronger signal of hot hand shooting than lower thresholds, they result in smaller sample sizes (see Miller and Sanjurjo (2014)). ${ }^{50}$

Here we repeat the analysis from Section 4.2, but with streak lengths of two, then four, rather than three. The results are summarized in Table A.3, alongside those of the primary analysis (indicated with a star) reported in Section 4.2. For each statistic, pooled effect sizes, along with the number of shooters that are above the 50th, and 95th, percentiles in terms of hot hand shooting are reported (with associated $p$-values).

With streak lengths set to two, the hit streak statistics $H_{F}$ and $H_{M}$ are on the hot side of the median for 27 ( $p=.004$, binomial test) and $29(p<.001)$ of the 37 primary shooters, respectively. ${ }^{51}$ They are each on the hot side of the 95 th percentile for 7 of the 37 shooters $(p=.002) .^{52}$ All average hit streak statistics remain highly statistically significant. Players on average shoot 6.6 percentage points better $(p<.001$, S.E. $=1.6 \mathrm{pp})$ when they have hit two or more shots in a row, as compared to any other recent shot history.

With streak lengths set to four, the hit streak statistics $H_{F}$ and $H_{M}$ are on the hot side of the median for 28 ( $p=.001$, binomial test)

\footnotetext{
${ }^{50}$ In the case of the conditional probability statistic that compares shooting performance when on a streak of hits to that following any other recent shot history, an additional consideration is that as the streak length becomes longer the measure gets increasingly conservative in the sense that it pits the improvement in performance when on longer streaks of hits directly against that when on shorter streaks of hits.

51 The length $H_{L}$ and runs $R$ statistics are unaffected by our choice of streak length.

52 Similarly, the minimum p-value across $H_{F}, H_{M}$, and $H_{L}$ is below the median of the corresponding minimum p-value sampling distribution for 31 of the 37 shooters $(p<.001)$, and below the 5 th percentile for 7 of the $37(p=.002)$.
} 
Table A.3

Robustness checks, separately varying streak lengths, permutation strata, and whether "warm-up" shots 1-3 in the round are excluded or not. Pooled effect sizes and counts of shooters, with associated p-values, for the relative improvement in shooting performance when on a streak of hits (Diff), the hit streak statistics for frequency $\left(H_{F}\right)$, momentum $\left(H_{M}\right)$, and length $\left(H_{L}\right)$, the minimum p-value across hit streak statistics $(M i n)$, and the runs statistic $(R)$. Count (binomial) tests are performed for the 50th and 95th percentiles of sampling distributions. For a visual representation of binomial tests on the hit streak statistics, while simultaneously varying streak lengths, permutation strata, and shooter inclusion criteria, see Figs. A.3-A.6.

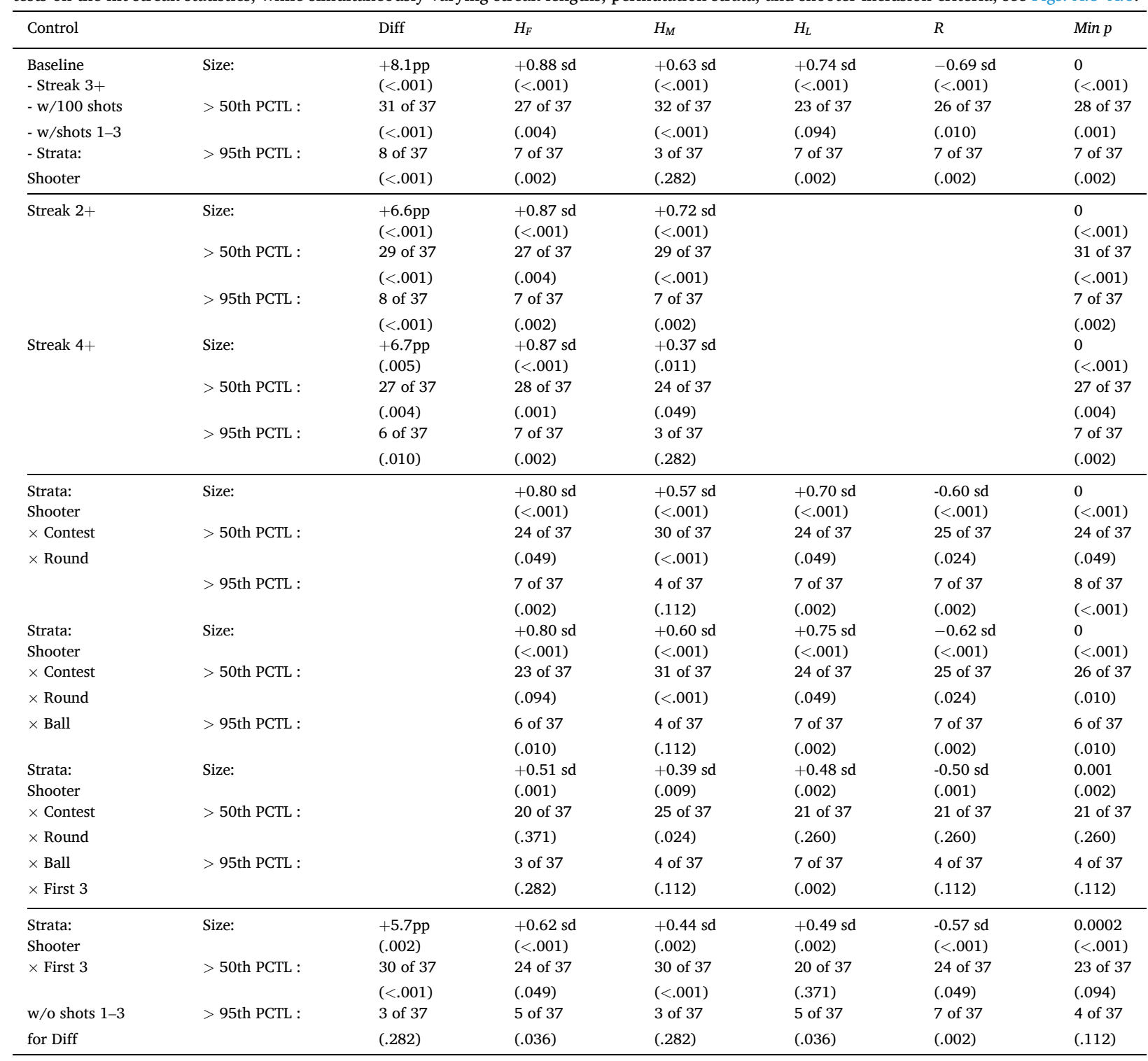

and $24(p=.049)$ of the 37 shooters, respectively. They are on the hot side of the 95th percentile for $7(p=.002)$ and $3(p=.282)$ of the 37 shooters, respectively. ${ }^{53}$ All average hit streak statistics remain highly statistically significant. Players on average shoot 6.7 percentage points better $(p=.005$, S.E. $=2.7 \mathrm{pp}$ ) when they have hit two or more shots in a row, as compared to any other recent shot history.

Thus, we see that the results from Section 4.2 are fairly robust to streak length, with a slight decrease in average performance when shooters are on a hit streak $(+8.1$ percentage points there), of around 1.5 percentage points.

\footnotetext{
53 Similarly, the minimum p-value across $H_{F}, H_{M}$, and $H_{L}$ is below the median of the corresponding minimum p-value sampling distribution for 27 of the 37 shooters $(p=.004)$, and below the 5 th percentile for 7 of the $37(p=.002)$.
} 


\section{Permutation Strata}

Choosing a permutation stratification scheme involves subtle but non-negligible trade-offs regarding the detection and interpretation of estimated hot hand effects.

In our primary analysis we follow the approach of Koehler and Conley (2003) by treating all of each shooter's shots as if they are taken under the same conditions, i.e. they belong to the same strata. A strength of this approach is that it can detect all systematic variations in shooter performance attributable to a hot hand that activates within rounds, between rounds, or even between contests. A potential weakness is that this allows for the possibility that systematic variations in shooting ability not due to the hot hand are misattributed to the hot hand. For example, this becomes possible if there is a steady improvement in shooter performance across rounds and contests. While this particular concern is mitigated by the fact that shooters show only a mild systematic tendency to improve across contests, and no such tendency across rounds (see Section 4.1), we now explore several alternative permutation stratification schemes and interpret the results.

In particular, we repeat the analysis from Section 4.2, but permuting shots within the following strata: (i) shooter $\times$ contest $\times$ round, (ii) shooter $\times$ contest $\times$ round $\times$ shot (ball) number on rack, and (iii) shooter $\times$ contest $\times$ round $\times$ shot number on rack with a separate strata for the first three shots in the round. The results are summarized in Table A.3, alongside those of the primary analysis (indicated with a star) reported in Section 4.2. For each statistic, pooled effect sizes, along with the number of shooters that are above the 50th, and 95th, percentiles in terms of hot hand shooting are reported (with associated p-values). As mentioned in Section 4.3, varying the permutation strata does not affect the estimated relative improvement in shooting performance when a shooter is on a streak of hits. Thus, we leave the corresponding column in Table A.3 empty. To see how the estimated relative improvement in shooting performance varies in a linear probability model with fixed effects corresponding to the different permutation strata, see the analysis under "Sensitivity of estimates to varying multiple features simultaneously" reported below, and Fig. A.7 in particular.

Permuting on the "shooter $\times$ contest $\times$ round" level, the hit streak and runs statistics $H_{F}, H_{M}, H_{L}$, and $R$ are on the hot side of the median for 24 ( $p=.049$, binomial test), $30(p<.001), 24(p=.049)$, and $25(p=.024)$ of the 37 shooters, respectively. They are on

\section{Table A.4}

Robustness checks, varying shooter inclusion criteria. Pooled effect sizes and counts of shooters, with associated p-values, for the relative improvement in shooting performance when on a streak of hits (Diff), the hit streak statistics for frequency $\left(H_{F}\right)$, momentum $\left(H_{M}\right)$, and length $\left(H_{L}\right)$, the minimum p-value across hit streak statistics (Min p), and the runs statistic $(R)$. Count (binomial) tests are performed for the 50th and 95th percentiles of sampling distributions. For a visual representation of binomial tests on the hit streak statistics, while simultaneously varying streak lengths, permutation strata, and shooter inclusion criteria, see Figs. A.3 - A.6.

\begin{tabular}{|c|c|c|c|c|c|c|c|}
\hline Control & & Diff & $H_{F}$ & $H_{M}$ & $H_{L}$ & $R$ & $\operatorname{Min} p$ \\
\hline $\begin{array}{l}\text { Baseline } \\
\text { - Streak } 3+ \\
\text { - w/100 shots }\end{array}$ & $\begin{array}{l}\text { Size: } \\
>\text { 50th PCTL : }\end{array}$ & $\begin{array}{l}+8.1 \mathrm{pp} \\
(<.001) \\
31 \text { of } 37\end{array}$ & $\begin{array}{l}+0.88 \mathrm{sd} \\
(<.001) \\
27 \text { of } 37\end{array}$ & $\begin{array}{l}+0.63 \mathrm{sd} \\
(<.001) \\
32 \text { of } 37\end{array}$ & $\begin{array}{l}+0.74 \text { sd } \\
(<.001) \\
23 \text { of } 37\end{array}$ & $\begin{array}{l}-0.69 \mathrm{sd} \\
(<.001) \\
26 \text { of } 37\end{array}$ & $\begin{array}{l}0 \\
(<.001) \\
28 \text { of } 37\end{array}$ \\
\hline $\begin{array}{l}\text { - w/shots } 1-3 \\
\text { - Strata: }\end{array}$ & > 95th PCTL : & $\begin{array}{l}(<.001) \\
8 \text { of } 37\end{array}$ & $\begin{array}{l}(.004) \\
7 \text { of } 37\end{array}$ & $\begin{array}{l}(<.001) \\
3 \text { of } 37\end{array}$ & $\begin{array}{l}(.094) \\
7 \text { of } 37\end{array}$ & $\begin{array}{l}(.010) \\
7 \text { of } 37\end{array}$ & $\begin{array}{l}(.001) \\
7 \text { of } 37\end{array}$ \\
\hline Shooter & & $(<.001)$ & $(.002)$ & $(.282)$ & $(.002)$ & $(.002)$ & $(.002)$ \\
\hline \multirow[t]{3}{*}{ w/125+ shots } & $\begin{array}{l}\text { Size: } \\
>\text { 50th PCTL : }\end{array}$ & $\begin{array}{l}+8.0 \mathrm{pp} \\
(<.001) \\
22 \text { of } 25\end{array}$ & $\begin{array}{l}+1.00 \mathrm{sd} \\
(<.001) \\
19 \text { of } 25\end{array}$ & $\begin{array}{l}+0.68 \mathrm{sd} \\
(<.001) \\
22 \text { of } 25\end{array}$ & $\begin{array}{l}+0.88 \mathrm{sd} \\
(<.001) \\
17 \text { of } 25\end{array}$ & $\begin{array}{l}-0.70 \mathrm{sd} \\
(<.001) \\
19 \text { of } 25\end{array}$ & $\begin{array}{l}0 \\
(<.001) \\
19 \text { of } 25\end{array}$ \\
\hline & > 95th PCTL : & $\begin{array}{l}(<.001) \\
6 \text { of } 25\end{array}$ & $\begin{array}{l}(.007) \\
5 \text { of } 25\end{array}$ & $\begin{array}{l}(<.001) \\
3 \text { of } 25\end{array}$ & $\begin{array}{l}(.054) \\
6 \text { of } 25\end{array}$ & $\begin{array}{l}(.007) \\
4 \text { of } 25\end{array}$ & $\begin{array}{l}(.007) \\
5 \text { of } 25\end{array}$ \\
\hline & & $(.001)$ & $(.007)$ & $(.127)$ & $(.001)$ & $(.034)$ & $(.007)$ \\
\hline \multirow[t]{5}{*}{$\mathrm{w} / 75+$ shots } & Size: & $\begin{array}{l}+6.5 p p \\
(<.001)\end{array}$ & $\begin{array}{l}+0.85 \mathrm{sd} \\
(<.001)\end{array}$ & $\begin{array}{l}+0.52 \mathrm{sd} \\
(<.001)\end{array}$ & $\begin{array}{l}+0.67 \mathrm{sd} \\
(<.001)\end{array}$ & $\begin{array}{l}-0.63 \mathrm{sd} \\
(<.001)\end{array}$ & $\begin{array}{l}0 \\
(<.001)\end{array}$ \\
\hline & $>$ 50th PCTL : & 34 of 46 & 32 of 46 & 35 of 46 & 28 of 46 & 32 of 46 & 34 of 46 \\
\hline & & $(<.001)$ & $(.006)$ & $(<.001)$ & $(.092)$ & $(.006)$ & $(<.001)$ \\
\hline & $>$ 95th PCTL : & 10 of 46 & 9 of 46 & 4 of 46 & 7 of 46 & 8 of 46 & 9 of 46 \\
\hline & & $(<.001)$ & $(<.001)$ & $(.197)$ & $(.008)$ & $(.002)$ & $(<.001)$ \\
\hline \multirow[t]{5}{*}{$\mathrm{w} / 1+$ shots } & Size: & $\begin{array}{l}+9.0 p p \\
(<.001)\end{array}$ & $\begin{array}{l}+0.50 \mathrm{sd} \\
(<.001)\end{array}$ & $\begin{array}{l}+0.34 \mathrm{sd} \\
(<.001)\end{array}$ & $\begin{array}{l}+0.41 \mathrm{sd} \\
(<.001)\end{array}$ & $\begin{array}{l}-0.42 \mathrm{sd} \\
(<.001)\end{array}$ & $\begin{array}{l}0 \\
(<.001)\end{array}$ \\
\hline & $>$ 50th PCTL : & 72 of 100 & 66 of 118 & 65 of 106 & 57 of 118 & 68 of 118 & 70 of 118 \\
\hline & & $(<.001)$ & $(.116)$ & $(.013)$ & $(.677)$ & $(.059)$ & $(.026)$ \\
\hline & $>$ 95th PCTL : & 17 of 100 & 14 of 118 & 8 of 106 & 12 of 118 & 12 of 118 & 17 of 118 \\
\hline & & $(<.001)$ & $(.002)$ & $(.161)$ & $(.015)$ & $(.015)$ & $(<.001)$ \\
\hline
\end{tabular}


the hot side of the 95th percentile for $7(p=.002), 4(p=.112), 7(p=.002)$, and $7(p=.002)$ of the 37 primary shooters, respectively. ${ }^{54}$ Each of the average hit streak and runs statistics remains highly statistically significant.

Permuting on the "shooter $\times$ contest $\times$ round $\times$ shot (ball) number on rack" level, the hit streak and runs statistics $H_{F}, H_{M}, H_{L}$, and $R$ are on the hot side of the median for 23 ( $p=.094$, binomial test), $31(p<.001), 24(p=.049)$, and 25 ( $p=.024)$ of the 37 shooters, respectively. They are on the hot side of the 95th percentile for $6(p=.010), 4(p=.112), 7(p=.002)$, and 7 ( $p=.002)$ of the 37 shooters, respectively. ${ }^{55}$ Each of the average hit streak and runs statistics remains highly statistically significant.

Permuting on the "shooter $\times$ contest $\times$ round $\times$ shot number on rack with a separate strata for the first three shots in the round" level, the hit streak and runs statistics $H_{F}, H_{M}, H_{L}$, and $R$ are on the hot side of the median for 20 ( $p=.371$, binomial test), 25 ( $p=$ $.024), 21$ ( $p=.260)$, and 21 ( $p=.260)$ of the 37 shooters, respectively. They are on the hot side of the 95th percentile for $3(p=.282)$, 4 ( $p=.112), 7(p=.002)$, and 4 ( $p=.112)$ of the 37 shooters, respectively. ${ }^{56}$ Each of the average hit streak and runs statistics remains highly statistically significant.

Thus, we see that the results from Section 4.2 are fairly robust even with many permutation strata, whereby the possibility of detecting hot hands that activate between contests, rounds, or even certain shot characteristics within the same round, is eliminated.

\section{Shooter inclusion criteria}

In Section 3 we discuss that the majority of shots taken in the history of the NBA three point contest (6287 of 9854) come from the minority of shooters that have taken at least 100 shots (37 of 123). Increasing the inclusion criterion to above 100 shots results in fewer shooters but less noisy hot hand effect estimates within those shooters. Reducing it does the opposite. In particular, it increases the proportion of shooters who have very few, or even zero, observations with which to compute the majority of our statistics.

We repeat the analysis from Section 4.2, but now including all shooters that have taken at least the following number of shots (rather than 100): (i) 125, (ii) 75, or (iii) 1. The results are summarized in Table A.4, alongside those of the primary analysis (indicated with a star) reported in Section 4.2. For each statistic, pooled effect sizes, along with the number of shooters that are above the 50th, and 95th, percentiles in terms of hot hand shooting are reported (with associated p-values).

Including all shooters that have taken at least 125 shots, the hit streak and runs statistics $H_{F}, H_{M}, H_{L}$, and $R$ are on the hot side of the median for 19 ( $p=.007$, binomial test), $22(p<.001), 17(p=.054)$, and $19(p=.007)$ of the 25 shooters, respectively. They are on the hot side of the 95th percentile for $5(p=.007), 3(p=.130), 6(p=.001)$, and $4(p=.034)$ of the 25 shooters, respectively. ${ }^{57}$ Each of the average hit streak and runs statistics remains highly statistically significant. Players on average shoot +8.0 percentage points better $(p<.001$, S.E. $=2.1 \mathrm{pp}$ ) when they have hit three or more shots in a row, as compared to any other recent shot history.

Including all shooters that have taken at least 75 shots, the hit streak and runs statistics $H_{F}, H_{M}, H_{L}$, and $R$ are on the hot side of the median for $32(p=.006$, binomial test), $35(p<.001), 28(p=.092)$, and $32(p=.006)$ of the 46 shooters, respectively. They are on the hot side of the 95th percentile for $9(p<.001), 4(p=.197), 7(p=.008)$, and $8(p=.002)$ of the 46 shooters, respectively. ${ }^{58}$ Each of the average hit streak and runs statistics remains highly statistically significant. Players on average shoot +6.5 percentage points better $(p<.001$, S.E. $=1.9 \mathrm{pp})$ when they have hit three or more shots in a row, as compared to any other recent shot history.

Including all shooters, the hit streak and runs statistics $H_{F}, H_{M}, H_{L}$, and $R$ are on the hot side of the median for 66 ( $p=.116$, binomial test), 65 ( $p=.156), 57(p=.677)$, and $68(p=.059)$ of the 118 shooters, respectively. They are on the hot side of the 95th percentile for $14(p=.002), 8(p=.238), 12(p=.015)$, and $12(p=.015)$ of the 118 shooters, respectively. ${ }^{59}$ Each of the average hit streak and runs statistics remains highly statistically significant. As reported in Section 4.2, the average improvement in shooting performance when shooters are on a streak of three or more hits (as compared to any other recent shot history), for all shooters with at least two instances of a shot following three or more hits, is $+9.0 \mathrm{pp}(p<.001$, S.E. $=2.3 \mathrm{pp}) .^{60}$

Thus, the prevalence and magnitude of hot hand shooting remains fairly consistent across the different inclusion criterion. As expected, as more low-volume shooters are included, their statistics scatter more randomly around the median. This drives corresponding binomial test $p$-values upwards. On the other hand, average shooting improvement when on a streak of hits remains fairly similar.

\footnotetext{
${ }^{54}$ Similarly, the minimum p-value across $H_{F}, H_{M}$, and $H_{L}$ is below the median of the corresponding minimum p-value sampling distribution for 24 of the 37 shooters $(p=.049)$, and below the 5 th percentile for 8 of the $37(p<.001)$.

55 Similarly, the minimum p-value across $H_{F}, H_{M}$, and $H_{L}$ is below the median of the corresponding minimum p-value sampling distribution for 26 of the 37 shooters $(p=.010)$, and below the 5th percentile for 6 of the $37(p=.010)$.

${ }^{56}$ Similarly, the minimum p-value across $H_{F}, H_{M}$, and $H_{L}$ is below the median of the corresponding minimum p-value sampling distribution for 23 of the 37 shooters $(p=.094)$, and below the 5 th percentile for 4 of the $37(p=.112)$.

${ }^{57}$ Similarly, the minimum $p$-value across $H_{F}, H_{M}$, and $H_{L}$ is below the median of the corresponding minimum p-value sampling distribution for 19 of the 25 shooters $(p=.007)$, and below the 5 th percentile for 5 of the $25(p=.007)$.

${ }^{58}$ Similarly, the minimum $p$-value across $H_{F}, H_{M}$, and $H_{L}$ is below the median of the corresponding minimum $p$-value sampling distribution for 34 of the 46 shooters $(p<.001)$, and below the 5 th percentile for 9 of the $46(p<.001)$.

${ }^{59}$ Similarly, the minimum $p$-value across $H_{F}, H_{M}$, and $H_{L}$ is below the median of the corresponding minimum $p$-value sampling distribution for 70 of the 118 shooters $(p=.026)$, and below the 5 th percentile for 17 of the $118(p<.001)$. Notice that 5 of the 123 shooters do not have at least one shot taken immediately following a streak of three or more hits, so the $H_{F}$ and $H_{M}$ statistics cannot be computed for them.

${ }^{60}$ At least two observations are necessary to compute standard errors for individual shooters. If we instead follow Koehler and Conley (2003), by only including all shooters that have at least five such instances, then the effect is $+8.9 \mathrm{pp}(p<.001$, S.E. $=2.0 \mathrm{pp})$.
} 


\section{Warm-up effect}

In Section 4.1 we report that shooters perform systematically worse on the first three shots of the round. In the most restrictive permutation test reported above we address this by creating a separate strata for these shots. The corresponding results are presented above.

While the validity of excluding the first three shots of the round is debatable, given that they may affect observer's hot hand beliefs, we report the results with these shots excluded in Table A.3, alongside those of the primary analysis (indicated with a star) reported in Section 4.2. For each statistic, pooled effect sizes, along with the number of shooters that are above the 50th, and 95th, percentiles in terms of hot hand shooting are reported (with associated p-values).

If we permute on the shooter level, with a separate strata for the first three shots in the round, the hit streak and runs statistics $H_{F}$, $H_{M}, H_{L}$, and $R$ are on the hot side of the median for 24 ( $p=.049$, binomial test), $30(p<.001), 20(p=.371)$, and 24 ( $\left.p=.049\right)$ of the

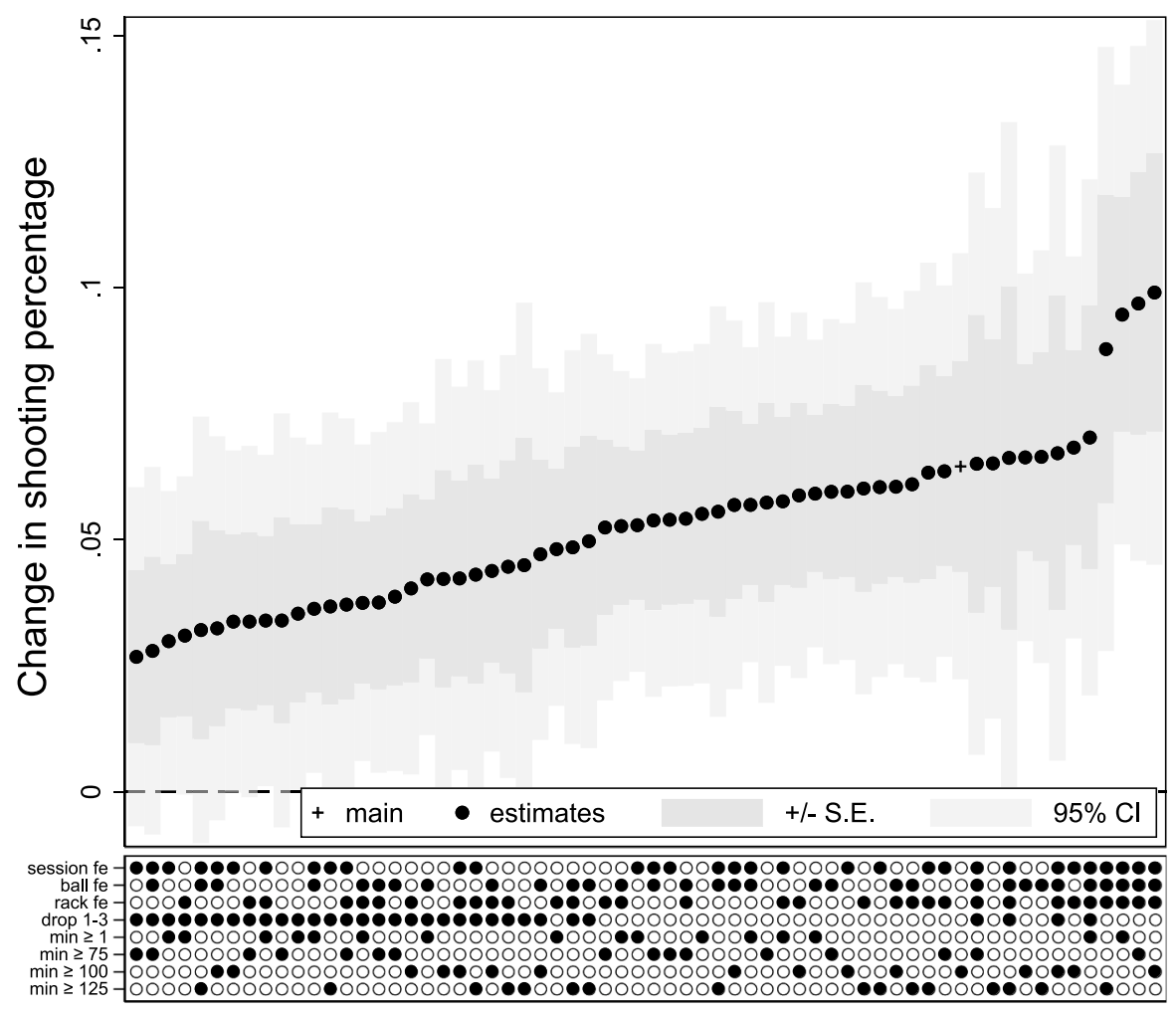

Fig. A.7. Shooters' (bias-adjusted) estimated improvement in performance (in percentage points) when on a streak of three or more hits, for 64 alternative specifications of a linear probability model that vary fixed effects ("fe"), whether the first three shots of the round are excluded, and the minimum number of shots required to include a shooter. The features of each specification (column) are indicated with black dots. Specifications are ordered from left to right in terms of increasing estimated effect size. 
37 shooters, respectively. They are on the hot side of the 95th percentile for $5(p=.036), 3(p=.282), 5(p=.036)$, and $7(p=.002)$ of the 37 shooters, respectively. ${ }^{61}$ Each of the average hit streak and runs statistics remains highly statistically significant.

With the first three shots of the round excluded, the increase in shooter performance is $+5.7(p=.002$, S.E. $=2.0 \mathrm{pp})$ percentage points when on a streak of three or more hits (as opposed to any other recent shot history), as compared to $+8.1 \mathrm{pp}$ when the first three shots of the round are included. Similar changes can be seen just below in Fig. A.7, where we estimate this effect size using a panel linear probability model under various combinations of different streak lengths, fixed effects, and inclusion criteria.

Sensitivity of estimates to varying multiple features simultaneously

In our primary analysis, presented in Section 4.2 , we report that the 37 primary shooters experience an estimated increase in shooting performance of +8.1 percentage points when on a streak of three or more hits.

As explained in Section 4.3, here we explore the sensitivity of the estimated improvement in shooting performance to a variety of alternative specifications. In Fig. A.7 we present the results using a specification curve (Simonsohn et al., 2019).

In particular, we estimate the improvement in shooting performance using a linear probability model with fixed effects, which we run on the unbalanced panel of shooters. We consider specifications that incrementally interact fixed effects for: (i) shooter ("sid"), (ii) contest $\times$ round ("session"), (iii) three rack locations: corner, wing, and center ("rack"), and (iv) order of the shot within the rack ("ball").

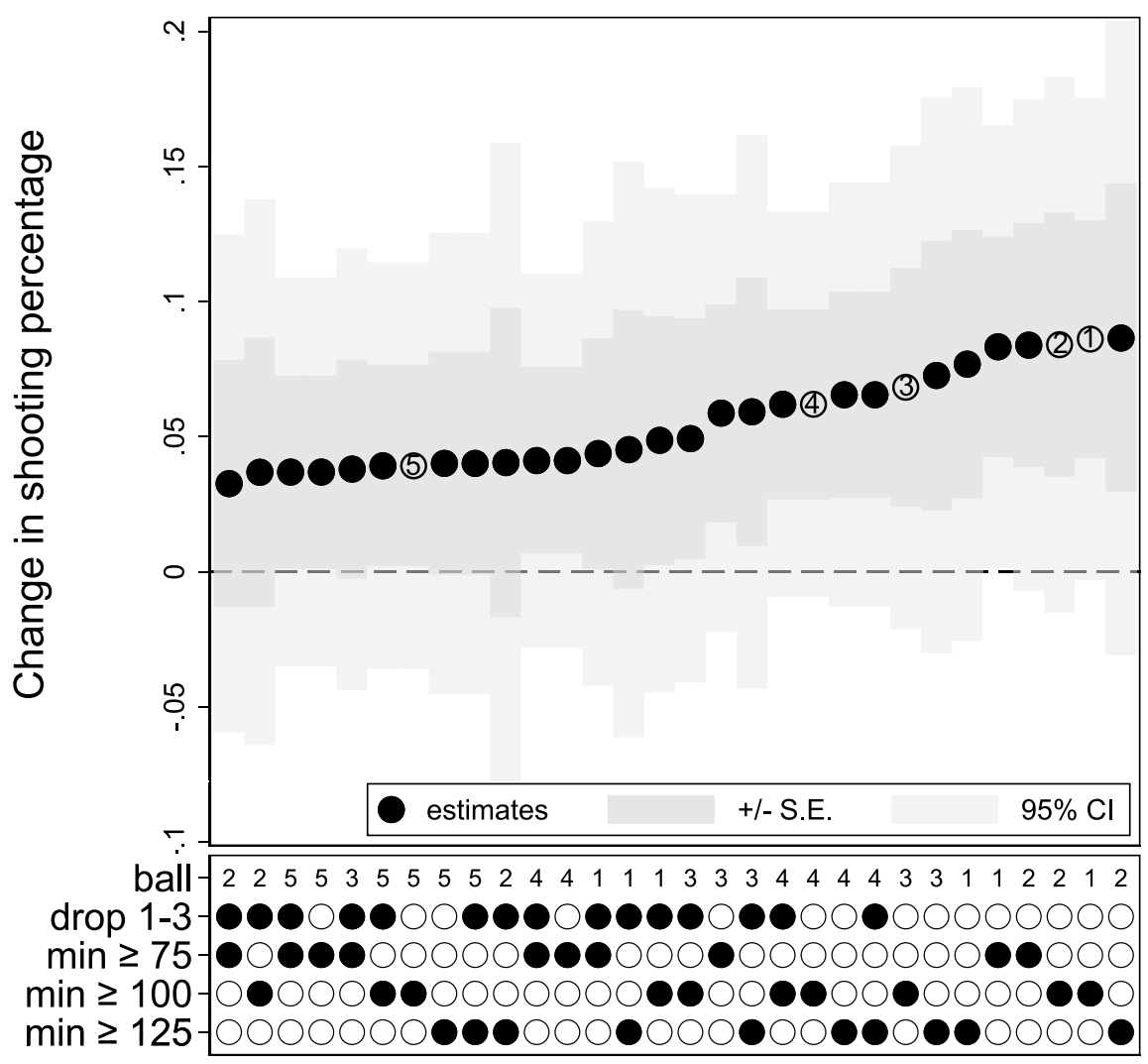

Fig. A.8. Shooters' estimated improvement in performance (in percentage points) when on a streak of three or more hits, for 30 alternative specifications of a linear probability model with shooter fixed effects, and that restrict shots to a particular ball order on the rack (1-5), whether the first three shots of the round are excluded, and the minimum number of shots required to include a shooter. The features of each specification (column) are indicated with black dots. Specifications are ordered from left to right in terms of increasing estimated effect size. The point estimate for each ball on the rack in the main specification is denoted by a hollow circle with the corresponding ball number inside of it.

\footnotetext{
61 The minimum p-value across $H_{F}, H_{M}$, and $H_{L}$ is below the median of the corresponding minimum p-value sampling distribution for 23 of the 37 shooters $(p=.094)$, and below the 5th percentile for 4 of the $37(p=.112)$.
} 
A benefit of adding more fixed effects is that consequent estimates will be insensitive to variation in shooting performance that is due solely to changes in shooting conditions. A weakness is that by the same token the model is not sensitive to a hot hand that may, for example, activate (at least partially) between rounds or contests. ${ }^{62}$

We specify the model as follows:

$$
\operatorname{hit}_{i s}=\alpha+\beta \text { hit }_{i s}+\boldsymbol{\gamma}_{i}^{\prime} \mathbf{I}+\epsilon_{i s}, \quad s \in S_{i}
$$

where hit $_{i s}$ is a dummy variable for whether player $i$ hit shot $s$, hit $3_{i s}$ is an indicator variable equal to one if player $i$ hit the three shots immediately preceding shot $s$, and $\gamma_{i}$ is a vector representing the shooter-specific fixed effect coefficients and their interactions.

We run a separate regression for each incremental interaction of the included fixed effects. In addition, we cross each of these alternative specifications with the four separate shooter inclusion criteria considered above (125, 100, 75, or 1 shots), and either drop or include the first three shots in the round to account for the "warm-up" effect described in Section 4.1. In total, this yields 64 alternative specifications of the model.

Fig. A.7 plots the (bias-adjusted) estimated effect for each of the specifications, along with associated standard error and 95 percent confidence interval. ${ }^{63}$ Dark circles directly beneath each plotted estimated effect indicate which fixed effects and inclusion criteria apply to the corresponding specification. The specifications are ordered from left to right in terms of increasing estimated effect size.

Overall, the estimated improvement in shooting performance is significantly larger than zero at the 5 percent level for 52 of the 64 specifications, and at the 10 percent level for 60 of the 64 . The minimum estimate of $+2.7 \mathrm{pp}(p=.118$, S.E. $=1.7 \mathrm{pp})$ corresponds to the specification with a session fixed effect, and that excludes: (i) shooters with fewer than 75 shots, and (ii) the first three shots of the round. The maximum estimate of $+9.9 \mathrm{pp}(p<.001$, S.E. $=2.8 \mathrm{pp})$ corresponds to the specification with fixed effects for session, rack, and ball, and that includes all shots and shooters with at least 100 shots. The estimate for the "main" specification, which is the one most comparable to the estimated average increase reported in Section 4.2, is $+6.5 p p(p=.004$, S.E. $=2.1 \mathrm{pp})$. The largest estimates, in general, correspond to specifications with more fixed effects and that include all shots from the round.

Note that the average effect size estimates from the unbalanced panel differ slightly from those of the corresponding raw unweighted effect sizes reported in Tables A.3 and A.4. For example, in our primary analysis the estimated effect size of the unbalanced panel is roughly $1.5 \mathrm{pp}$ less than the raw unweighted average effect of $+8.1 \mathrm{pp}$. This is because the players with more shots in the history of the contest are more heavily weighted in the coefficient estimate of the unbalanced panel, and happen to produce a smaller (though not significantly smaller) hot hand effect. In terms of interpreting these different estimates, on the one hand the raw unweighted effect size is roughly representative of the hot hand effect that we would observe from a shooter drawn at random from the sample. On the other hand, the weighted estimate is representative of the hot hand effect we would observe on a shot that is randomly drawn from the dataset. That is, it puts greater weight on the performance of shooters that viewers see more of-so could have a greater impact on the formation of hot hand beliefs.

\section{Sensitivity of estimates, by ball on rack}

We estimate the impact of a streak of three or more hits on subsequent shooting performance, separately for each ball on the rack, $i$. e. the racks' first ball, second ball,..., fifth ball. As above, we use a linear probability model with shooter fixed effects. While restricting each such estimate of the hot hand to 20 percent of the data in this way yields relatively noisier estimates, it provides us a rough indication of the sensitivity of hot hand effect size to the shot (ball) number on rack.

We consider various specifications, in which in addition to running a separate regression for each of the 5 balls on the rack, we either include or exclude the first three shots in the round, and include all shooters that have taken at least 75, 100, or 125 shots. By considering all possible combinations, we get a total of 30 specifications. ${ }^{64}$ The estimated improvement in shooting performance when on a streak of hits for each of these specifications is presented in Fig. A.8, along with standard error and 95 percent confidence interval. Specifications are ordered from left to right in terms of increasing estimated effect size. ${ }^{65}$

The figure shows clearly that estimated improvement in shooting performance does not increase systematically with the ball on the rack. In fact, it tends to do the opposite. This can be seen clearly, for example, with the main specification, whose estimates for each ball on the rack are denoted by hollow circles with the corresponding number enclosed inside.

\section{Supplementary material}

Supplementary material associated with this article can be found, in the online version, at 10.1016/j.euroecorev.2021.103771

\footnotetext{
${ }^{62}$ For example, a player may be more confident in his shooting ability going into the contest in one year than in another, or his confidence level might increase in between the rounds of a contest based on new observed information, such as seeing his opponents shoot poorly.

${ }^{63}$ Each estimated $\widehat{\beta}$ is bias-adjusted, using simulated shot outcomes in which we set each player's hit probability equal to his observed shooting percentage.

${ }^{64}$ Given that each specification considers just 1 of the 5 balls on the racks we set the minimal inclusion criteria at 75 shots. Similarly, we refrain from adding additional fixed effects, and thus thinning out the data further. In any case, adding session fixed effects yields similar estimates, though with larger standard errors.

${ }^{65}$ These estimated improvements do not require bias adjustments because in each specification there is no overlap between streaks of hits and the dependent variable (see Miller and Sanjurjo (2018)).
} 


\section{References}

Aharoni, G., Sarig, O.H., 2011. Hot hands and equilibrium. Appl. Econ. 44 (18), 2309-2320. https://doi.org/10.1080/00036846.2011.564141.

Arkes, J., 2010. Revisiting the hot hand theory with free throw data in a multivariate framework. J. Quant. Anal. Sports 6.

Arkes, J., 2011. Do gamblers correctly price momentum in nba betting markets? J. Predict. Mark. 5 (1), 31-50.

Arkes, J., 2013. Misses in 'hot hand' research. J. Sports Econ. 14 (4), 401-410. https://doi.org/10.1177/1527002513496013.

Attali, Y., 2013. Perceived hotness affects behavior of basketball players and coaches. Psychol. Sci. 24 (7), 1151-1156.

Avery, C., Chevalier, J., 1999. Identifying investor sentiment from price paths: the case of football betting. J. Bus. 72 (4), $493-521$.

Avugos, S., Bar-Eli, M., Ritov, I., Sher, E., 2013. The elusive reality of efficacy performance cycles in basketball shooting: analysis of players' performance under invariant conditions. Int. J. Sport Exer. Psychol. 11, 184-202.

Bandura, A., 1982. Self-efficacy mechanism in human agency. Am. Psychol. 37 (2), 122-147.

Bocskocsky, A., Ezekowitz, J., Stein, C., 2014. The hot hand: a new approach to an old 'fallacy'. 8th Annual Mit Sloan Sports Analytics Conference.

Barberis, N., Thaler, R., 2003. A survey of behavioral finance. Princeton University Press. 1, 1053-1128.

Brown, W.A., Sauer, R.D., 1993. Does the basketball market believe in the hot hand? comment. Am. Econ. Rev. 83 (5), $1377-1386$.

Camerer, C.F., 1989. Does the basketball market believe in the 'hot hand,'? Am. Econ. Rev. 79 (5), $1257-1261$.

Cao, Z., 2011. Essays on Behavioral Economics. Oregon State University.

Carlson, K.A., Shu, S.B., 2007. The rule of three: how the third event signals the emergence of a streak. Org. Behav. Hum. Decis. Process. 104 (1), $113-121$.

Churchland, M.M., Afshar, A., Shenoy, K.V., 2006. A central source of movement variability. Neuron 52, $1085-1096$.

Croson, R., Sundali, J., 2005. The gambler's fallacy and the hot hand: empirical data from casinos. J. Risk Uncertain. 30 (3), $195-209$.

Csikszentmihalyi, M., 1988. The flow experience and its significance for human psychology. In: Csikszentmihalyi, M., Csikszentmihalyi, I.S. (Eds.), Optimal

Experience: Psychological Studies of Flow in Consciousness. Cambridge University, New York, NY, US.

De Bondt, W.P., 1993. Betting on trends: intuitive forecasts of financial risk and return. Int. J. Forecast. 9 (3), $355-371$.

De Long, J.B., Shleifer, A., Summers, L.H., Waldmann, R.J., 1991. The survival of noise traders in financial-markets. J. Bus. 64 (1), 1-19.

Durham, G.R., Hertzel, M.G., Martin, J.S., 2005. The market impact of trends and sequences in performance: new evidence. J. Financ. 60 (5), $2551-2569$.

Suetens, S., Galbo-Jørgensen, C.B., Tyran, J.-R., 2016. Predicting lotto numbers: a natural experiment on the gambler's fallacy and the hot-hand fallacy. J. Eur. Econ. Assoc. 14 (3), 584-607.

Gilovich, T., Vallone, R., Tversky, A., 1985. The hot hand in basketball: on the misperception of random sequences. Cogn. Psychol. $17,295-314$.

Gould, S.J., 1989. The streak of streaks. Chance 2 (2), 10-16.

Green, B., Zwiebel, J., 2018. The hot-hand fallacy: Cognitive mistakes or equilibrium adjustments? Evidence from major league baseball. Manag. Sci. 64 (11), 5315-5348.

Guryan, J., Kearney, M.S., 2008. Gambling at lucky stores: empirical evidence from state lottery sales. Am. Econ. Rev. 98 (1), $458-473$.

Kahneman, D., 1973. Attention and Effort. Prentice Hall.

Kahneman, D., 2011. Thinking, fast and slow. Farrar, Straus and Giroux.

Kahneman, D., Riepe, M.W., 1998. Aspects of investor psychology: beliefs, preferences, and biases investment advisors should know about. J. Portf. Manag. 24 (4), $1-21$.

Koehler, J.J., Conley, C.A., 2003. The "hot hand" myth in professional basketball. J. Sport Exer. Psychol. 25 (2), $253-259$.

Lee, M., Smith, G., 2002. Regression to the mean and football wagers. J. Behav. Decis. Mak. 15 (4), 329-342.

Loh, R.K., Warachka, M., 2012. Streaks in earnings surprises and the cross-section of stock returns. Manag. Sci. 58 (7), 1305-1321. https://doi.org/10.1287/ mnsc.1110.1485.

MacKinnon, J.G., 2009. Bootstrap hypothesis testing. Handbook of computational econometrics 183, 213.

Malkiel, B.G., 2011. A Random Walk Down Wall Street: The Time-Tested Strategy for Sucessful Investing. W. W. Norton \& Company, New York.

Miller, J. B., Sanjurjo, A., 2014. A cold shower for the hot hand fallacy: Robust evidence that belief in the hot hand is justified. Working Paper. Available at https://osf. io/pj79r/.

Miller, J.B., Sanjurjo, A., 2018. Surprised by the hot hand fallacy? a truth in the law of small numbers. Econometrica 86 (6), $2019-2047$.

Nalebuff, B., 1987. Puzzles: choose a curtain, duel-ity, two point conversions, and more. J. Econ. Perspect. $157-163$.

Narayanan, S., Manchanda, P., 2012. An empirical analysis of individual level casino gambling behavior. Quant. Mark. Econ. 10 (1), 27-62. https://doi.org/10.1007/ s11129-011-9110-7.

Neiman, T., Loewenstein, Y., 2011. Reinforcement learning in professional basketball players. Nat. Commun. 2:569.

Paul, R.J., Weinbach, A.P., 2005. Bettor misperceptions in the nba: the overbetting of large favorites and the 'hot hand'. J. Sports Econ. 6 (4), 390-400. https://doi. org/10.1177/1527002504266861.

Rabin, M., Vayanos, D., 2010. The gambler's and hot-hand fallacies: theory and applications. Rev. Econ. Stud. 77, 730-778.

Rao, J. M., 2009a. Experts' perceptions of autocorrelation: the hot hand fallacy among professional basketball players. Working Paper.

Rao, J. M., 2009b. When the gambler's fallacy becomes the hot hand fallacy: an experiment with experts and novices. Working Paper.

Reifman, A., 2012. Hot Hand: The Statistics Behind Sports' Greatest Streaks. Potomac Books.

Simonsohn, U., Simmons, J.P., Nelson, L.D., 2019. Specification curve: descriptive and inferential statistics on all reasonable specifications. Available at SSRN 2694998.

Sinkey, M., Logan, T., 2013. Does the hot hand drive the market? Eastern Econ. J. https://doi.org/10.1057/eej.2013.33. Advance online publication.

Smith, G., Levere, M., Kurtzman, R., 2009. Poker player behavior after big wins and big losses. Manag. Sci. 55 (9), 1547-1555. https://doi.org/10.1287/ mnsc.1090.1044.

Stone, D.F., 2012. Measurement error and the hot hand. Am. Stat. 66 (1), 61-66.

Sundali, J., Croson, R., 2006. Biases in casino betting: the hot hand the gambler's fallacy. Judg. Decis. Mak. 1 (1), 1-12.

Thaler, R.H., Sunstein, C.R., 2008. Nudge: Improving Decisions about Health, Wealth, and Happiness. Yale University Press.

Tversky, A., Gilovich, T., 1989. The cold facts about the "hot hand" in basketball. Chance 2 (1), 16-21.

Wardrop, R.L., 1995. Simpson'S paradox and the hot hand in basketball. Am. Stat. 49 (1), 24-28.

Wardrop, R. L., 1999. Statistical tests for the hot-hand in basketball in a controlled setting. Unpublished manuscript 1, 1-20.

Westfall, P.H., Young, S.S., 1993. Resampling-based Multiple Testing: Examples and Methods for p-value Adjustment, 279. John Wiley \& Sons.

Xu, J., Harvey, N., 2014. Carry on winning: the gambler's fallacy creates hot hand effects in online gambling. Cognition 131 (2), 173-180. https://doi.org/10.1016/j. cognition.2014.01.002.

Yaari, G., Eisenmann, S., 2011. The hot (invisible?) hand: can time sequence patterns of success/failure in sports be modeled as repeated random independent trials? PLoS One 6 (10), 1-10.

Yuan, J., Sun, G.Z., Siu, R., 2014. The lure of illusory luck: how much are people willing to pay for random shocks. J. Econ. Behav. Org. 106, 269-280. 